\title{
Infinity structure of Poincaré duality spaces
}

\author{
THOMAS TRADLER \\ MAHMOUD ZEINALIAN \\ ApPendiX A By DenNis Sullivan
}

\begin{abstract}
We show that the complex $C_{\bullet} X$ of rational simplicial chains on a compact and triangulated Poincaré duality space $X$ of dimension $d$ is an $A_{\infty}$ coalgebra with $\infty$ duality. This is the structure required for an $\mathrm{A}_{\infty}$ version of the cyclic Deligne conjecture. One corollary is that the shifted Hochschild cohomology $\mathrm{HH}^{\bullet+d}\left(\mathrm{C}^{\bullet} \mathrm{X}, \mathrm{C} \bullet \mathrm{X}\right)$ of the cochain algebra $C^{\bullet} X$ with values in $C_{\bullet} X$ has a BV structure. This implies, if $X$ is moreover simply connected, that the shifted homology $H_{\bullet+d} L X$ of the free loop space admits a BV structure. An appendix by Dennis Sullivan gives a general local construction of $\infty$ structures.
\end{abstract}

57P10; 57P05

\section{Introduction}

The Hochschild complex of an associative algebra with an invariant, symmetric, and nondegenerate inner product has a natural action of the PROP of Sullivan chord diagrams by work of the authors [25]. This was referred to as the cyclic Deligne conjecture. Unfortunately, such a statement does not directly apply to the topology of Poincaré duality spaces, since the associative algebra of simplicial cochains does not enjoy a nondegenerate and symmetric inner product which is also invariant. As it turns out, the right notion for this cyclic Deligne conjecture is that of an $\mathrm{A}_{\infty}$ coalgebra with $\infty$ duality; that is, an $\mathrm{A}_{\infty}$ coalgebra $C$ together with a duality map $F$ enjoying appropriate invariance, nondegeneracy, and symmetry properties; see Definition 2.3.2. This structure was defined by the first author [22], where it was shown that the Hochschild cohomology $H H^{\bullet+d}\left(C^{*}, C\right)$ is a BV algebra. In fact, we later showed in [24] that the PROP $\mathcal{D} \mathcal{G}_{2}$, an enhancement of the PROP of Sullivan chord diagrams, acts on this Hochschild complex $C H^{\bullet+d}\left(C^{*}, C\right)$. In this paper, we give an explicit construction of an $\infty$ duality structure on $C_{\bullet} X$, the rational chains on a compact and triangulated Poincaré duality space $X$ of dimension $d$. This implies the shifted Hochschild cohomology $H H^{\bullet+d}\left(C^{\bullet} X, C_{\bullet} X\right)$ of the cochains $C^{\bullet} X$ with values in $C_{\bullet} X$ has a natural BV structure with a unit. A corollary of this, when $X$ is in addition simply connected, is 
that the shifted homology $H_{\bullet+d} L X$ of the free loop space admits a unital BV structure. This should be viewed in light of Chas and Sullivan's seminal work on the algebraic topology of the free loop space $L X$, when $X$ is a manifold [1].

Let us recall some of the history and motivations. Chas and Sullivan [1] defined a BV structure on the shifted homology $H_{\bullet+d} L X$ of the free loop space $L X$ of an orientable manifold $X$, with a unit only in the closed case; see Remark 1.0.3. More precisely, they defined a multiplication

$$
\therefore H_{\bullet+d} L X \otimes H_{\bullet+d} L X \rightarrow H_{\bullet+d} L X
$$

called the loop product, along with an operator

$$
\Delta: H_{\bullet+d} L X \rightarrow H_{\bullet+1+d} L X,
$$

and proved that the triple $\left(H_{\bullet+d} L X, ., \Delta\right)$ forms a BV algebra. That is to say that $\left(H_{\bullet+d} L X,.\right)$ is an associative and graded commutative algebra with a unit along with a differential $\Delta$ whose deviation from being a derivation defines a structure of a graded Lie algebra on $H_{\bullet+d+1} L X$. The multiplication - arises from the fact that two loops with common base points can be composed and that in a manifold cycles can be made transversal and then intersected. They coined the term string topology to describe the activity aimed towards understanding the algebraic structure of the free loop space of a manifold. Since then, there have been several generalizations and applications of their machinery in different directions; see, for instance, Sullivan [21], Chas and Sullivan [2], Cohen, Klein and Sullivan [5], Cohen and Jones [4], Klein [10], Felix, Thomas and Vigue-Poirrier [26], Merkulov [17] and Chataur [3].

Remark 1.0.1 Extension of string topology to more general spaces than manifolds have been considered by others. For example, J Klein [10] defined a product on the loop space homology $H_{\bullet} L X$ for an orientable Poincaré duality space $X$. In the case of a manifold, this product coincides with that of string topology.

Remark 1.0.2 The Chas-Sullivan BV structure is in fact a homotopy invariant of the manifold [5]. This fits with the work of this paper as well as with the view that the Chas-Sullivan BV structure is defined for a lager category of spaces than manifolds. Nonetheless, Sullivan conjectures that the further string topology, involving operations labelled by the rest of moduli space of Riemann surfaces, is not a homotopy invariant; see Conjecture 3 in the Postscript of [21]. 
A corollary of the result of this paper is that there is a unital BV structure on the shifted cohomology of the larger class of simply connected, compact, triangulated Poincaré duality spaces. By definition, a Poincaré duality space $X$ of dimension $d$ is a topological space with an element $[X] \in H_{d} X$, called the fundamental class, such that the map $[X] \cap \cdot: H^{\bullet} X \rightarrow H_{d-\bullet} X$ is an isomorphism. A subtle but very important point is that although the above map is a map of $H^{\bullet} X$ bimodules, when viewed at the chain level as a map $C^{\bullet} X \rightarrow C_{d-\bullet} X$, it is not a map of $C^{\bullet} X$-bimodules. A closed, oriented manifold is a good example of a Poincaré duality space and it is therefore natural to ask if in this case our structure coincides with the Chas-Sullivan BV structure. This question, however, has not been addressed in this paper.

Let us highlight some of the salient points of this paper. We have used an inductive and combinatorial argument to construct a symmetric $\mathrm{A}_{\infty}$ co-inner product, and subsequently used minimal models to obtain an $\infty$ duality structure. As a result we show the following:

(i) The chain complex $C \cdot X$ on a compact and triangulated Poincaré duality space $X$ is naturally an $\mathrm{A}_{\infty}$ coalgebra with $\infty$ duality.

(ii) For $X$ as in (i), the shifted Hochschild cohomology $H H^{\bullet+d}\left(C^{\bullet} X, C \bullet X\right)$ is a BV algebra with a unit; see Remark 1.0.3.

(iii) If $X$ is in addition simply connected, then the shifted homology $H_{\bullet+d} L X$ of the free loop space is naturally a BV algebra with a unit.

Remark 1.0.3 Whether or not the Chas-Sullivan loop multiplication has a unit is decided by whether or not the manifold in question is closed, ie compact and without boundary. This suggests there should be a version of the construction of this paper without a unit which works for the larger category of Lefschetz duality spaces.

A general and very useful scheme for the local construction of $\infty$ structures is provided by Dennis Sullivan in Appendix A. In Appendix B, an explicit calculation of the $\mathrm{A}_{\infty}$ co-inner product for the case of the circle shows the necessity of having higher homotopies even when the $\mathrm{A}_{\infty}$ coalgebra structure is in fact strictly coassociative. Such an explicit calculation of the structure in higher dimensions, even for the case of a two-dimensional compact and orientable surface, should be very interesting.

Let us review the content of each chapter in more details. In Section 2, we present the algebraic definitions necessary for the future chapters. An $\mathrm{A}_{\infty}$ coalgebra structure over a graded module $C$ is a differential on the free associative algebra $B C$ over the shifted vector space $C$; see Tradler [19]. An $\mathrm{A}_{\infty}$ cobimodule over an $\mathrm{A}_{\infty}$ coalgebra is defined in a similar fashion by having a differential satisfying certain properties in terms of 
its interaction with the differential of the $\mathrm{A}_{\infty}$ coalgebra over which it is defined; see Markl [14]. An $\infty$ duality is a map $F$ between $\mathrm{A}_{\infty}$ cobimodule $C$ and its dual $C^{*}$, which satisfies a symmetry condition and induces an isomorphism at the homology level; see Tradler [23]. We recall from [22] that an $\infty$ duality structure on $C$ yields a BV structure on the Hochschild cohomology $\mathrm{HH}^{\bullet}\left(C^{*}, C\right)$. In Section 3, we show that the chains $C_{\bullet} X$ on a compact polyhedron $X$ of dimension $d$, together with a choice of a cycle $\mu \in C_{\bullet} X$, is naturally furnished with a locally constructed $\mathrm{A}_{\infty}$ co-inner product structure satisfying a symmetry condition; see Proposition 3.1.3. Moreover, we show that when $X$ is a Poincaré duality space and $\mu$ is its fundamental class $[X]$, this symmetric $\mathrm{A}_{\infty}$ co-inner product is an $\infty$ duality structure; that is, the $\mathrm{A}_{\infty}$ co-inner product structure induces an isomorphism at the level of the homology; see Theorem 3.1.4. This follows from Theorem 2.2.5 which is proved in two steps. First, we argue that an $\mathrm{A}_{\infty}$ module over an $\mathrm{A}_{\infty}$ algebra has a minimal model; see Section 2.2. Then, using methods similar to those employed by Sullivan [20] and Gómez-Tato, Halperin and Tanré [7], we show that an $\mathrm{A}_{\infty}$ module map between two minimal models which induces an isomorphism at the level of the homology is in fact an isomorphism. Algebraically, this inverse map can be written down very explicitly. It is noteworthy that even though the $\infty$ duality map is local, there is no guarantee for the locality of the algebraically constructed inverse map; see Remark 3.1.5. Nonetheless, such a structure induces a quasi-isomorphism between the Hochschild complexes $\mathrm{CH}^{\bullet}\left(\mathrm{C}^{\bullet} \mathrm{X}, \mathrm{C}^{\bullet} \mathrm{X}\right)$ and $C H^{\bullet}\left(C^{\bullet} X, C \bullet X\right)$. According to [22] the transport of the algebraic structures from one Hochschild cohomology onto the other, and putting everything together, after a shift in degrees, yields a BV structure. A result of Jones [8] states that, for a simply connected space $X$, the Hochschild cohomology $H H^{\bullet}\left(C^{\bullet} X, C \bullet X\right)$ calculates the homology $H_{\bullet} L X$ of the free loop space. Therefore, it follows that if the Poincare duality space $X$ is in addition simply connected, the shifted homology $H_{\bullet+d} L X$ of the free loop space $L X$ is naturally a BV algebra. There are two appendices to this paper. Appendix A, written by Dennis Sullivan, provides a general local construction for infinity structures. In a second appendix, we study the $\infty$ duality structure on C. $S^{1}$, the chains on the circle $S^{1}$. This structure, in terms of its component maps, is explicitly calculated.

Acknowledgements We would like to thank Dennis Sullivan for adding an appendix to this paper and for his continuous support and suggestions. We would like to thank Andrew Ranicki and Ralph Cohen for useful discussions and Scott Wilson for pointing out a gap in an earlier version. We are also thankful for the referee's comments which have improved the presentation of the paper. 


\section{Algebraic definitions}

\section{$2.1 \infty$ duality structure}

In this section, we recall some of the pertinent algebraic and homotopy notions. The Poincaré duality constitutes a $H^{\bullet} X$-bimodule isomorphism between cohomology $H^{\bullet} X$ and homology $H_{d-\bullet} X$ of a Poincaré duality space $X$ of dimension $d$. The correct homotopy notion is obtained by resolving the operad of bimodule maps.

Remark 2.1.1 By Longoni and Tradler [13], given a cyclic quadratic operad $\mathcal{O}$, there exists a colored operad $\widehat{\mathcal{O}}$ so that an algebra over $\widehat{\mathcal{O}}$ is precisely an algebra over $\mathcal{O}$ together with a symmetric and invariant inner product. Furthermore, the Koszulness of $\mathcal{O}$ as an operad implies Koszulness of $\widehat{\mathcal{O}}$ as a colored operad. More precisely, let $\mathcal{O}^{!}$denote the quadratic dual operad of $\mathcal{O}$ and $\mathbf{D}\left(\mathcal{O}^{!}\right)$its cobar dual operad. Also, let $\hat{\mathcal{O}}^{!}$be the quadratic dual colored operad of $\widehat{\mathcal{O}}$, whose cobar dual colored operad is denoted by $\mathbf{D}\left(\widehat{\mathcal{O}}^{!}\right)$. The Koszulness of $\mathcal{O}$ means that the canonical map $\mathbf{D}\left(\mathcal{O}^{!}\right) \rightarrow \mathcal{O}$ is a quasi-isomorphism of operads. This implies that the canonical map $\mathbf{D}\left(\widehat{\mathcal{O}}^{!}\right) \rightarrow \widehat{\mathcal{O}}$ is also a quasi-isomorphism of colored operads; see [13, Theorem 2.8]. This statement allows one to resolve $\widehat{\mathcal{O}}$ to obtain a homotopy version $\widehat{\mathcal{O}}_{\infty}=\mathbf{D}\left(\widehat{\mathcal{O}}^{!}\right)$of both the algebra and the inner product.

We now apply the result from [13] to the cyclic quadratic Koszul operad Assoc which governs associative algebras. Let us describe the outcome of the construction $\widehat{\mathcal{A s s o c}}_{\infty}$ more explicitly. Let $C=\bigoplus_{j \in \mathbb{Z}} C_{j}$ be a graded module over fixed associative and commutative ring $R$ with a unit. Define the suspension $s C$ of $C$ as the graded module $s C=\bigoplus_{j \in \mathbb{Z}}(s C)_{j}$ with $(s C)_{j}=C_{j+1}$. The suspension map $s: C \rightarrow s C, v \mapsto s c=c$ is a linear isomorphism of degree -1 . We denote by $B C=T(s C)=\prod_{i \geq 0}(s C)^{\otimes i}$ the (completed) free associative algebra on the suspended space $s C$.

An $\mathrm{A}_{\infty}$ coalgebra structure on $C$ is a derivation $D \in \operatorname{Der}(B C)$ of degree +1 with $D^{2}=0$. The space $\operatorname{Der}(B C)$ has a differential $\delta: \operatorname{Der}(B C) \rightarrow \operatorname{Der}(B C)$ given by $\delta\left(D^{\prime}\right)=\left[D, D^{\prime}\right]=D \circ D^{\prime}-(-1)^{\left|D^{\prime}\right|} D^{\prime} \circ D$ with $\delta^{2}=0$. Given an $A_{\infty}$ coalgebra $(B C, D)$ and a graded $R$-module $M$, let

$$
B^{M} C=T^{s M}(s C)=\prod_{i, j \geq 0}(s C)^{\otimes i} \otimes(s M) \otimes(s C)^{\otimes j} .
$$

An $\mathrm{A}_{\infty}$ cobimodule structure on $M$ over $C$ is a derivation $D^{M} \in \operatorname{Der}\left(B^{M} C\right)$ over $D$ of degree +1 with $\left(D^{M}\right)^{2}=0$ [23].

For $\mathrm{A}_{\infty}$ cobimodules $\left(B^{M} C, D^{M}\right)$ and $\left(B^{N} C, D^{N}\right)$ over the $\mathrm{A}_{\infty}$ coalgebra $B C$, let $\operatorname{Hom}\left(B^{M} C, B^{N} C\right)$ denote the space of all module maps. Hom $\left(B^{M} C, B^{N} C\right)$ 
has a natural differential given by $\delta^{M, N}(F)=D^{N} \circ F-(-1)^{|F|} F \circ D^{M}$. An $\mathrm{A}_{\infty}$ cobimodule map between $B^{M} C$ and $B^{N} C$ is by definition an element $F$ in $\operatorname{Hom}\left(B^{M} C, B^{N} C\right)$ of degree 0 with $\delta^{M, N}(F)=0$; that is, $D^{N} \circ F=F \circ D^{M}$. Note that since $F$ is a map between completed free objects, it is uniquely determined by the components $\left.F\right|_{M}: M \rightarrow B^{N} C$.

Remark 2.1.2 The concepts of $\mathrm{A}_{\infty}$ coalgebras, $\mathrm{A}_{\infty}$ cobimodules, and $\mathrm{A}_{\infty}$ cobimodule maps are generalizations of those of coassociative coalgebras, cobimodules, and cobimodule maps [23].

Consider an $\mathrm{A}_{\infty}$ cobimodule map $F: B^{M} C \rightarrow B^{N} C$. We will argue that the induced map $F_{*}: H_{\bullet}\left(B^{M} C, D^{M}\right) \rightarrow H_{\bullet}\left(B^{N} C, D^{N}\right)$, between the homologies, is an isomorphism if and only if the lowest component $F_{0,0}: M \rightarrow N$ of $F$ induces an isomorphism $\left(F_{0,0}\right)_{*}: H_{\bullet}\left(M, D_{0,0}^{M}\right) \rightarrow H_{\bullet}\left(N, D_{0,0}^{N}\right)$ between the homologies. Such a map is called a quasi-isomorphism. We show this using by using a minimal model decomposition for $\mathrm{A}_{\infty}$ modules.

\subsection{Minimal models and invertibility}

Every free connected differential graded algebra is isomorphic to the tensor product of a unique minimal algebra and a unique contractible algebra [20]. Since, similar decomposition theorems have been proved and utilized in different contexts; for instance see Gómez-Tato, Halperin and Tanré [7, Section 8], Kajiura [9, Theorem 5.2], and the Lemma in Kontsevich [11, Section 4.5.1]. In this section, we prove that every $\mathrm{A}_{\infty}$ cobimodule $\left(B^{M} C, D^{M}\right)$ over $(B C, D)$ can be decomposed into a direct sum of a linear contractible and a minimal one. This will allow us to show that an $\mathrm{A}_{\infty}$ cobimodule map $F: B^{M} A \rightarrow B^{N} A$ induces an isomorphism on homology $F_{*}: H_{\bullet}\left(B^{M} A, D^{M}\right) \rightarrow H_{\bullet}\left(B^{N} A, D^{N}\right)$, if the lowest component $F_{0,0}: M \rightarrow N$ induces an isomorphism on homology $\left(F_{0,0}\right)_{*}: H_{\bullet}\left(M, D_{0,0}^{M}\right) \rightarrow H_{\bullet}\left(N, D_{0,0}^{N}\right)$. For similar a statement in a different context, see Kajiura [9, Theorem 5.4]. For a more general operadic method see Markl [15, (M3)].

Definition 2.2.1 Let $(B C, D)$ be an $\mathrm{A}_{\infty}$ coalgebra and $\left(B^{P} C, D^{P}\right)$ be an $\mathrm{A}_{\infty}$ cobimodule. $\left(B^{P} C, D^{P}\right)$ is called minimal, if the lowest component $D_{0,0}^{P}: P \rightarrow P$ of the map $D^{P}: B^{P} C \rightarrow B^{P} C$ vanishes, ie $D_{0,0}^{P}=0$.

Definition 2.2.2 Let $(B C, D)$ be an $\mathrm{A}_{\infty}$ coalgebra and $\left(B^{N} C, D^{N}\right)$ be an $\mathrm{A}_{\infty}$ cobimodule. $\left(B^{N} C, D^{N}\right)$ is called linear contractible, if the lowest component of $D^{N}: B^{N} C \rightarrow B^{N} C$ is the only nonvanishing component, ie for $i+j>0$ it is $D_{i, j}^{N}=0$, and additionally the homology of $\left(N, D_{0,0}^{N}\right)$ vanishes. 
With this we can state the decomposition theorem.

Theorem 2.2.3 For $(B C, D)$ an $A_{\infty}$ coalgebra, every $A_{\infty}$ cobimodule $\left(B^{M} C, D^{M}\right)$ is isomorphic as an $A_{\infty}$ cobimodule to the direct sum of a minimal one $\left(B^{P} C, D^{P}\right)$ and a linear contractible one $\left(B^{N} C, D^{N}\right)$. That is,

$$
B^{M} C \cong B^{P} C \oplus B^{N} C \text {. }
$$

Proof Choose an arbitrary positive definite inner product on the vector space $M$. Denote by $d$ the lowest component of the differential on $B^{M} C$, ie $d=D_{0,0}^{M}$. Define $d^{\dagger}$ to be the adjoint of $d$ under the chosen inner product. Then, define $X=\operatorname{Im}(d)$, $Y=\operatorname{Im}\left(d^{\dagger}\right)$, and the harmonic subspace $P=\operatorname{Ker}(d) \cap \operatorname{Ker}\left(d^{\dagger}\right)$. One knows that $M$ has a Hodge decomposition $M=P \oplus X \oplus Y$. We will use the decomposition $M=P \oplus N$, where $N=X \oplus Y$. An elementary check shows that $d: Y \rightarrow X$ is an isomorphism of vector spaces and that every homology class $[m]$ of $(M, d)$ has a unique harmonic representative.

We want to show that there exists a sequence $\left\{\left(B^{P \oplus N} C, D^{P \oplus N}(n)\right)\right\}_{n \in \mathbb{N}_{0}}$ of $A_{\infty}$ cobimodules structures on the space $B^{P \oplus N} C$, whose components will be written as $\left.D^{P \oplus N}(n)\right|_{P \oplus N}=d_{0}^{n}+d_{1}^{n}+d_{2}^{n}+\cdots$, where

$$
d_{j}^{n}: P \oplus N \rightarrow\left(B^{P \oplus N} C\right)_{j}=\bigoplus_{r+s=j} C^{\otimes r} \otimes(P \oplus N) \otimes C^{\otimes s} .
$$

These maps will satisfy

$$
\begin{array}{lll}
d_{0}^{n}(x)=0 & d_{0}^{n}(y) \in X & d_{0}^{n}(p)=0, \\
d_{k}^{n}(x)=0 & d_{k}^{n}(y)=0 & d_{k}^{n}(p) \in\left(B^{P} C\right)_{k},
\end{array}
$$

for $x \in X, y \in Y, p \in P$, and $1 \leq k \leq n$, together with the compatibility condition

$$
d_{k}^{n}=d_{k}^{m}, \quad \text { for } \quad k \leq \min (m, n) .
$$

Furthermore, there are $A_{\infty}$ cobimodules isomorphisms

$$
\varphi(n):\left(B^{M} C, D^{M}\right) \rightarrow\left(B^{P \oplus N} C, D^{P \oplus N}(n)\right)
$$

satisfying the compatibility,

$$
\varphi_{k}^{n}=\varphi_{k}^{m}, \quad \text { for } \quad k \leq \min (m, n),
$$

where $\varphi_{k}^{n}$ is the component of $\left.\varphi(n)\right|_{M}=\varphi_{0}^{n}+\varphi_{1}^{n}+\varphi_{2}^{n}+\cdots$ that maps to $B^{(P \oplus N)} C_{k}$. After constructing these maps and structures, we obtain an $A_{\infty}$ cobimodule structure on $B^{(P \oplus N)} C$ by taking components $\left(D^{P \oplus N}\right)_{k}=d_{k}^{n}$ for any $n \geq k$. Using equation (2-1) and (2-2), it is clear that this splits into a minimal $A_{\infty}$ cobimodule $\left(B^{P} C, D^{P}\right)$ 
and a linear contractible one $\left(B^{N} C, D^{N}\right)$. Furthermore the maps $\varphi(n)$ induce an $A_{\infty}$ cobimodule isomorphism $\varphi: B^{M} C \rightarrow B^{P} C \oplus B^{N} C=B^{(P \oplus N)} C$ by $\varphi_{k}=\varphi_{k}^{n}$, for $n \geq k$.

Let us start with the case $n=0$. We identify $B^{P \oplus N} C=B^{M} C$, and take $D^{P \oplus N}(0)=$ $D^{M}$ together with $\varphi(0)=\mathrm{id}: B^{M} C \rightarrow B^{P \oplus N} C$. Then, condition (2-1) is satisfied, because we have $d_{0}^{0}=d$, and with the definitions of $X, Y$ and $P$ it is clear that $d(X)=d(P)=\{0\}$, while $d(Y) \subset X$.

Now, assume we have constructed $D^{P \oplus N}(k)$ and $\varphi(k)$ for $k=1, \cdots, n$, satisfying (2-1) and (2-2). Then, define a tensor-bimodule isomorphism $F: B^{P \oplus N} C \rightarrow B^{P \oplus N} C$ to be given by $\left.F\right|_{P \oplus N}=\mathrm{id}+f$. The map $f: P \oplus N \rightarrow\left(B^{P \oplus N} C\right)_{n+1}$ is given by

$$
\begin{array}{ll}
f(x)=-d_{n+1}^{n}\left(\left(d_{0}^{n}\right)^{-1}(x)\right), & \text { for } \quad x \in X \\
f(y)=0, & \text { for } \quad y \in Y \\
f(p) \text { satisfies } \widetilde{d}_{0}^{n}(f(p))=(\mathrm{id}-\pi) \circ d_{n+1}^{n}(p), & \text { for } \quad p \in P
\end{array}
$$

where $d_{0}^{n}=d: Y \rightarrow X$, and $\pi$ is the projection $\pi: B^{M} C \rightarrow B^{P} C$. In order to see that $f(p)$ can be defined, first notice that

$$
0=\left(D^{P \oplus N}(n)\right)^{2}(p)=\widetilde{d}_{0}^{n} \circ d_{n+1}^{n}(p)+\sum_{j=1}^{n+1} \widetilde{d}_{j}^{n} \circ d_{n+1-j}^{n}(p) .
$$

By the inductive hypotheses (2-1) and (2-2), we have $\widetilde{d}_{0}^{n} \circ d_{n+1}^{n}(p) \in B^{N} C$ and $\sum_{j=1}^{n+1} \widetilde{d_{j}^{n}} \circ d_{n+1-j}^{n}(p) \in B^{P} C$, so that $\widetilde{d}_{0}^{n} \circ d_{n+1}^{n}(p)=0$. Furthermore, it is also the case that $\widetilde{d}_{0}^{n} \circ \pi \circ d_{n+1}^{n}(p)=\pi \circ \widetilde{d}_{0}^{n} \circ d_{n+1}^{n}(p)=0$. Thus, (id $\left.-\pi\right) \circ d_{n+1}^{n}(p) \in B^{N} C$ is a closed element. But $H_{\bullet}\left(B^{N} C, \widetilde{d}_{0}^{n}\right)=\cdots \otimes H_{\bullet}\left(N,\left.d\right|_{N}\right) \otimes \cdots=\{0\}$, so one can find an element $f(p) \in B^{N} C$ such that $\widetilde{d}_{0}^{n}(f(p))=(\mathrm{id}-\pi) \circ d_{n+1}^{n}(p)$.

Thus, $F$ defines a vector space isomorphism with inverse given by $\left.F^{-1}\right|_{P \oplus N}=$ id $-f+$ higher order terms. With this, we define $D^{P \oplus N}(n+1)$ to be the induced $A_{\infty}$ cobimodule structure of $F$ on $B^{P \oplus N} C$, ie $D^{P \oplus N}(n+1)=F \circ D^{P \oplus N}(n) \circ F^{-1}$. This defines an $A_{\infty}$ cobimodule structure and we get an $A_{\infty}$ cobimodule isomorphism by taking $\varphi(n+1)=F \circ \varphi(n)$. Notice that $D^{P \oplus N}(n+1)=F \circ D^{P \oplus N}(n) \circ F^{-1}=$ $(\mathrm{id}+f) \circ\left(d_{0}^{n}+d_{1}^{n}+\cdots\right) \circ(\mathrm{id}-f+\cdots)$, so that $d_{k}^{n+1}=d_{k}^{n}$ for $k \leq n$, and $d_{n+1}^{n+1}=$ $d_{n+1}^{n}+f \circ d_{0}^{n}-d_{0}^{n} \circ f$. Similarly $\varphi(n+1)=F \circ \varphi(n)=(\mathrm{id}+f) \circ\left(\varphi_{0}^{n}+\varphi_{1}^{n}+\cdots\right)$ implies $\varphi_{k}^{n+1}=\varphi_{k}^{n}$ for $k \leq n$. Since the differential $D^{P \oplus N}(n)$ and the isomorphism $\varphi(n)$ are not altered up to the $n$-th level, only equation (2-2) with $k=n+1$ requires 
a check. Recall that $d_{0}^{n}=d_{0}^{0}=d$, so that for $x \in X=\operatorname{Im}(d)$, we have

$$
\begin{aligned}
d_{n+1}^{n+1}(x) & =d_{n+1}^{n}(x)+f\left(d_{0}^{n}(x)\right)-\widetilde{d}_{0}^{n}(f(x)) \\
& =d_{n+1}^{n}(x)+\widetilde{d}_{0}^{n}\left(d_{n+1}^{n}\left(\left(d_{0}^{n}\right)^{-1}(x)\right)\right) \\
& =d_{n+1}^{n}(x)-d_{n+1}^{n}\left(d_{0}^{n}\left(\left(d_{0}^{n}\right)^{-1}(x)\right)\right) \\
& =0 .
\end{aligned}
$$

Note that we made use of the fact that $\left(D^{P \oplus N}(n)\right)^{2}(y)=0$, for $y \in Y$, implies $0=d_{n+1}^{n} \circ d_{0}^{n}(y)+\widetilde{d}_{n}^{n} \circ d_{1}^{n}(y)+\cdots+\widetilde{d}_{0}^{n} \circ d_{n+1}^{n}(y)=d_{n+1}^{n} \circ d_{0}^{n}(y)+\widetilde{d}_{0}^{n} \circ d_{n+1}^{n}(y)$, because $d_{1}^{n}(y)=d_{2}^{n}(y)=\cdots=d_{n}^{n}(y)=0$ by condition (2-2).

Next, for any $y \in Y$, we have

$$
\begin{aligned}
d_{n+1}^{n+1}(y) & =d_{n+1}^{n}(y)+f\left(d_{0}^{n}(y)\right)-\widetilde{d}_{0}^{n}(f(y)) \\
& =d_{n+1}^{n}(y)-d_{n+1}^{n}\left(\left(d_{0}^{n}\right)^{-1}\left(d_{0}^{n}(y)\right)\right) \\
& =0,
\end{aligned}
$$

because $d_{0}^{n}(y) \in X$.

Finally, if $p \in P$, we have

$$
\begin{aligned}
d_{n+1}^{n+1}(p) & =d_{n+1}^{n}(p)+f\left(d_{0}^{n}(p)\right)-\widetilde{d}_{0}^{n}(f(p)) \\
& =d_{n+1}^{n}(p)-(\mathrm{id}-\pi) \circ d_{n+1}^{n}(p) \\
& =\pi \circ d_{n+1}^{n}(p) \in B^{P} C .
\end{aligned}
$$

Definition 2.2.4 Let $(B C, D)$ be an $\mathrm{A}_{\infty}$ coalgebra. Given an $\mathrm{A}_{\infty}$ cobimodule morphism $F:\left(B^{M_{1}} C, D^{M_{1}}\right) \rightarrow\left(B^{M_{2}} C, D^{M_{2}}\right)$ between two $\mathrm{A}_{\infty}$ cobimodules, $F$ is called a quasi-isomorphism if the lowest component $F_{0,0}: M_{1} \rightarrow M_{2}$ induces an isomorphism on homology:

$$
\left(F_{0,0}\right)_{*}: H_{\bullet}\left(M_{1}, D_{0,0}^{M_{1}}\right) \cong H_{\bullet}\left(M_{2}, D_{0,0}^{M_{2}}\right) .
$$

Theorem 2.2.5 Let $(B C, D)$ be an $A_{\infty}$ coalgebra. If $F:\left(B^{M} C, D^{M}\right) \rightarrow\left(B^{N} C, D^{N}\right)$ is a map of $A_{\infty}$ cobimodules which is a quasi-isomorphism, then there is an $A_{\infty}$ cobimodule morphism $G:\left(B^{N} C, D^{N}\right) \rightarrow\left(B^{M} C, D^{M}\right)$ so that $G_{*} \circ F_{*}=\operatorname{id}_{H_{\bullet}\left(B^{M} C, D^{M}\right)}$ and $F_{*} \circ G_{*}=\operatorname{id}_{H_{\bullet}\left(B^{N} C, D^{N}\right)}$.

Proof For $i=1,2$, decompose $B^{M_{i}} C=B^{P_{i}} C \oplus B^{N_{i}} C$ into a direct sum of a minimal and a linear contractible $\mathrm{A}_{\infty}$ cobimodules. Consider the projection

$$
\operatorname{pr}^{i}:\left(B^{M_{i}} C, D^{M_{i}}\right) \rightarrow\left(B^{P_{i}} C, D^{P_{i}}\right)
$$


and the inclusion

$$
\text { incl }^{i}:\left(B^{P_{i}} C, D^{P_{i}}\right) \rightarrow\left(B^{M_{i}} C, D^{M_{i}}\right) .
$$

Since $H_{*}\left(B^{N_{i}} C, D^{N_{i}}\right)=\{0\}$, the maps $\mathrm{pr}^{i}$ and incl ${ }^{i}$ induce isomorphisms between the homologies of $\left(B^{M_{i}} C, D^{M_{i}}\right)$ and $\left(B^{P_{i}} C, D^{P_{i}}\right)$. Let $\Phi=\mathrm{pr}^{2} \circ F \circ$ incl $^{1}$.

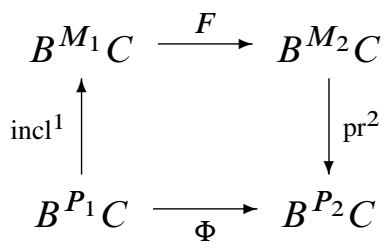

Note that $\Phi_{0,0}:\left(P_{1}, 0\right) \rightarrow\left(P_{2}, 0\right)$ induces an isomorphism, after passing to the homology. Therefore, since the complexes $\left(P_{1}, 0\right)$ and $\left(P_{2}, 0\right)$ have zero differentials, $\Phi_{0,0}$ is in fact an isomorphism. We claim that $\Phi$ has a right inverse $\rho$, which can be constructed inductively to satisfy $\Phi \circ \rho=$ id. Define $\rho_{0,0}=\left(\Phi_{0,0}\right)^{-1}$. Note that the $(k, l)$-th level equation $\Phi_{0,0} \circ \rho_{k, l}+$ (lower terms in $\left.\rho_{r, s}\right)=$ id implies that $\rho_{k, l}=\left(\Phi_{0,0}\right)^{-1} \circ(\mathrm{id}-\cdots)$. Therefore, $\rho_{k, l}$ 's can be inductively solved. Thus, a right inverse $\rho$ is constructed. Similarly, one can construct the left inverse $\lambda$. Thus, $\Phi$ is has the inverse $\rho=\lambda$. Let $G=\operatorname{incl}^{1} \circ \rho \circ \mathrm{pr}^{2}$. By passing to the homology, we have

$$
\begin{aligned}
& \operatorname{id}_{H_{*}\left(B^{M_{1}} C, D^{M_{1}}\right)}=\left(\mathrm{incl}^{1}\right)_{*} \circ\left(\mathrm{pr}^{1}\right)_{*} \\
& =\left(\mathrm{incl}^{1}\right)_{*} \circ \rho_{*} \circ \Phi_{*} \circ\left(\mathrm{pr}^{1}\right)_{*} \\
& =\left(\mathrm{incl}^{1}\right)_{*} \circ \rho_{*} \circ\left(\mathrm{pr}^{2}\right)_{*} \circ F_{*} \circ\left(\mathrm{incl}^{1}\right)_{*} \circ\left(\mathrm{pr}^{1}\right)_{*} \\
& =G_{*} \circ F_{*} \text {. }
\end{aligned}
$$

Similarly, we have

$$
\operatorname{id}_{H_{*}\left(B^{\left.M_{2} C, D^{M_{2}}\right)}\right.}=F_{*} \circ G_{*} .
$$

\subsection{Complex of $\mathrm{A}_{\infty}$ co-inner products}

We will now introduce a complex suitable for dealing with $\mathrm{A}_{\infty}$ co-inner products. By construction, an $\mathrm{A}_{\infty}$ co-inner product will be a closed element in this complex.

Given graded modules $V, W, X$, and $Y$ over a ring $R$, let

$$
T_{Y}^{X} V=\prod_{k, l \geq 0} V^{\otimes k} \otimes X \otimes V^{\otimes l} \otimes Y .
$$

The components are written as the products $T_{Y}^{X} V_{k, l}=V^{\otimes k} \otimes X \otimes V^{\otimes l} \otimes Y$ and $T_{Y}^{X} V_{n}=\bigoplus_{k+l=n} V^{\otimes k} \otimes X \otimes V^{\otimes l} \otimes Y$. For any map $\mathcal{X}: W \rightarrow T_{Y}^{X} V$, denote the 
component of $\mathcal{X}$ mapping to $T_{Y}^{X} V_{n}$ by $\mathcal{X}_{n}$; that is,

$$
\mathcal{X}_{n}=\operatorname{pr}_{T_{Y}^{X} V_{n}} \circ \mathcal{X}: W \stackrel{\mathcal{X}}{\rightarrow} T_{Y}^{X} V \stackrel{\mathrm{pr}}{\rightarrow} T_{Y}^{X} V_{n} .
$$

Let $(B C, D)$ be an $\mathrm{A}_{\infty}$ coalgebra. Notice that an $\mathrm{A}_{\infty}$ co-inner product is determined by a map $C^{*} \rightarrow B^{C} C$. Clearly, every element $F \in T_{C}^{C} C$ gives such a map by dualizing the last tensor factor. We work with the grading and differential $\mathcal{D}$ on $T_{C}^{C} C$ making the inclusion incl: $\left(T_{C}^{C} C, \mathcal{D}\right) \hookrightarrow\left(\operatorname{Hom}\left(C^{*}, B^{C} C\right), \delta^{C^{*}, C}\right)$ a chain map of degree zero. To be consistent with previous notation, we use $B_{C}^{C} C$ for the space $T_{C}^{C} C$ with this correct grading. Therefore, the inclusion incl: $\left(B_{C}^{C} C, \mathcal{D}\right) \hookrightarrow\left(\operatorname{Hom}\left(C^{*}, B^{C} C\right), \delta^{C^{*}, C}\right)$ is a chain map of degree 0 with $\delta^{C^{*}, C}(\operatorname{Im}($ incl $)) \subset \operatorname{Im}($ incl $)$.

Notice that $\mathrm{A}_{\infty}$ co-inner products are exactly the closed elements of the complex $\left(\operatorname{Hom}\left(C^{*}, B^{C} C\right), \delta^{C^{*}, C}\right)$ of degree zero. Thus, every closed element of $\left(B_{C}^{C} C, \mathcal{D}\right)$ of degree zero determines an $\mathrm{A}_{\infty}$ co-inner product. More explicitly, the degree of an element

$$
F=\left(c_{1} \otimes \cdots \otimes c_{n}\right) \otimes c^{\prime} \otimes\left(c_{1}^{\prime \prime} \otimes \cdots \otimes c_{m}^{\prime \prime}\right) \otimes c^{\prime \prime \prime} \in(C)^{\otimes n} \otimes C \otimes(C)^{\otimes m} \otimes C
$$

with $c_{i} \in C_{k_{i}}, c^{\prime} \in C_{k^{\prime}}, c_{i}^{\prime \prime} \in C_{k_{i}^{\prime \prime}}$, and $c^{\prime \prime \prime} \in C_{k^{\prime \prime \prime}}$ is given by

$$
\|F\|=\sum_{i=1}^{n}\left(k_{i}-1\right)+\left(k^{\prime}-1\right)+\sum_{i=1}^{m}\left(k_{i}^{\prime \prime}-1\right)+\left(1-k^{\prime \prime \prime}\right) .
$$

The differential $\mathcal{D}: B_{C}^{C} C \rightarrow B_{C}^{C} C$ is given by a sum obtained by applying $D$ at all possible places in $B_{C}^{C} C$ in a cyclic way [23]:

$$
\begin{aligned}
\mathcal{D} & \left(c_{1}, \cdots, c_{n}, c_{n+1}, c_{n+2}, \cdots, c_{n+m+1}, c_{n+m+2}\right) \\
& =\sum_{1 \leq i \leq n} \pm\left(\cdots, c_{i-1}, D\left(c_{i}\right), c_{i+1}, \cdots\right) \pm\left(\cdots, c_{n}, D^{C}\left(c_{n+1}\right), c_{n+2}, \cdots\right) \\
& +\sum_{n+2 \leq i \leq n+m+1} \pm\left(\cdots, c_{i-1}, D\left(c_{i}\right), c_{i+1}, \cdots\right) \pm \sigma\left(\cdots, c_{n+m+1}, D^{C}\left(c_{n+m+2}\right)\right),
\end{aligned}
$$

where

$$
\sigma: C^{\otimes r} \otimes C \otimes C^{\otimes s} \otimes C \otimes C^{\otimes t} \rightarrow C^{\otimes t+r} \otimes C \otimes C^{\otimes s} \otimes C=B_{C}^{C} C_{t+r, s}
$$

is a cyclic rotation of elements. As usual, the signs are determined by the Koszul sign rule, which says whenever an element of degree $p$ moves over an element of degree $q$, a sign of $(-1)^{p q}$ is introduced. Let us use a diagrammatic picture for $\mathcal{D}$ described in [23]. If we draw the two special $C$ components of $B_{C}^{C} C$ on the horizontal axis, then the differential can be pictured in the following way: 


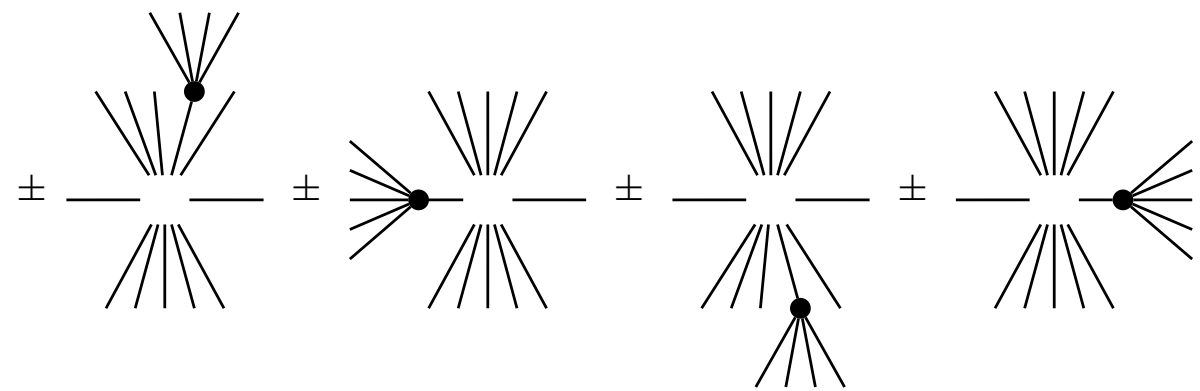

(The only difference between the above and [23] is that here we apply all of $D=$ $D_{1}+D_{2}+\cdots$ including the differential $D_{1}$, and not just the higher terms.) Let us recall why $\mathcal{D}^{2}=0$ [23]. The diagrams for $\mathcal{D}^{2}$ are given by applying $D$ at two places. There are two cases. Either one of the two multiplications is placed on top of the other, in which case $D^{2}=0$ shows that the sum of those diagrams vanish. Or, the multiplications are placed at different positions in which case each term appears twice with opposite signs and therefore cancel out each other.

Given a map $f: B C \rightarrow B C^{\prime}$ between two $\mathrm{A}_{\infty}$ coalgebras $(B C, D)$ and $\left(B C^{\prime}, D^{\prime}\right)$, one can define $\hat{f}: B_{C}^{C} C \rightarrow B_{C^{\prime}}^{C^{\prime}} C^{\prime}$ by taking a sum over all possibilities of applying $f$ at all possible places simultaneously. More precisely, $\hat{f}$ on $C^{\otimes n} \otimes C \otimes C^{\otimes m} \otimes C$ is given by

$$
\begin{aligned}
& \hat{f}\left(c_{1}, \cdots, c_{n}, c_{n+1}, c_{n+2}, \cdots, c_{n+m+1}, c_{n+m+2}\right)= \\
& \sum_{r, s, t, u} \pm \sigma\left(f\left(c_{1}\right), \cdots, f\left(c_{n}\right), i^{r, s}\left(f\left(c_{n+1}\right)\right), f\left(c_{n+2}\right), \cdots,\right. \\
& \left.f\left(c_{n+m+1}\right), i^{t, u}\left(f\left(c_{n+m+2}\right)\right)\right),
\end{aligned}
$$

where $i^{r, s}: C^{\otimes r+s+1} \stackrel{\cong}{\rightarrow} C^{\otimes r} \otimes C \otimes C^{\otimes s}$ is the canonical isomorphism and $\sigma$ is the cyclic rotation (2-3). In other words, we have to take the same cyclic rules for the positions of the elements $c_{i}$ that were taken in the definition of $\mathcal{D}$. After applying $f$ in all spots simultaneously, we need to determine the two special $C$ components. This is done by taking a sum of all possibilities of special components which come from the two original special components

and

$$
\begin{aligned}
c_{n+1} & \mapsto \sum_{k \geq 1} \sum_{r+s+1=k} i^{r, s}\left(f_{k}\left(c_{n+1}\right)\right) \\
c_{n+m+2} & \mapsto \sum_{k \geq 1} \sum_{t+u+1=k} i^{t, u}\left(f_{k}\left(c_{n+m+2}\right)\right) .
\end{aligned}
$$


In order to be an element of $B_{C^{\prime}}^{C^{\prime}} C^{\prime}$, the last factor in the tensor product has to be one of the special $C$ components. Therefore, it might be necessary to apply a cyclic rotation $\sigma$. Diagrammatically, we have the following:

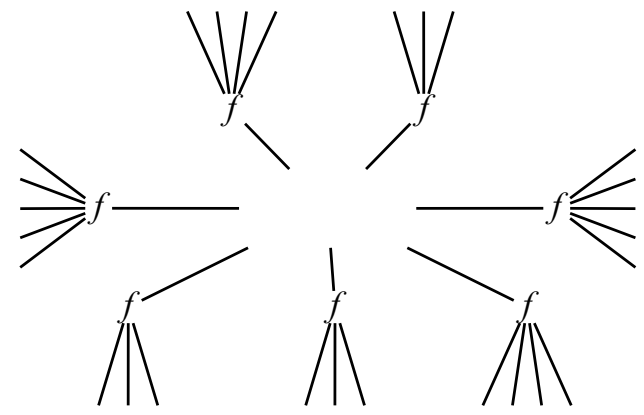

Proposition 2.3.1 $\hat{f}:\left(B_{C}^{C} C, \mathcal{D}\right) \rightarrow\left(B_{C^{\prime}}^{C^{\prime}} C^{\prime}, \mathcal{D}^{\prime}\right)$ is a chain map.

Proof $\mathcal{D}^{\prime} \circ \hat{f}$ corresponds to applying $f$ and having exactly one multiplication $D^{\prime}$ outside the ring of $f$ 's; see above picture. $\hat{f} \circ \mathcal{D}$ corresponds to applying $f$ and having exactly one multiplication $D$ inside the ring of $f$ 's. But the fact that $f$ is an $\mathrm{A}_{\infty}$ algebra map $\left(D^{\prime} \circ f=f \circ D\right)$ means exactly that $f$ commutes with inside and outside multiplication of $D^{\prime}$ and $D$.

Let us recall the concept of symmetry from [22] and [13], and its application to the Hochschild complex as stated in [22].

Let $\tau: B_{C}^{C} C \rightarrow B_{C}^{C} C$ denote the map which rotates the tensor factors cyclically by $180^{\circ}$; that is,

$$
\begin{gathered}
\tau: C^{\otimes n} \otimes C \otimes C^{\otimes m} \otimes C \rightarrow C^{\otimes m} \otimes C \otimes C^{\otimes n} \otimes C, \\
\left(c_{1} \otimes \cdots \otimes c_{n}\right) \otimes c^{\prime} \otimes\left(c_{1}^{\prime \prime} \otimes \cdots \otimes c_{m}^{\prime \prime}\right) \otimes c^{\prime \prime \prime} \mapsto \\
(-1)^{\epsilon}\left(c_{1}^{\prime \prime} \otimes \cdots \otimes c_{m}^{\prime \prime}\right) \otimes c^{\prime \prime \prime} \otimes\left(c_{1} \otimes \cdots \otimes c_{n}\right) \otimes c^{\prime},
\end{gathered}
$$

where $\epsilon=\left(\left|c_{1}\right|+\cdots+\left|c_{n}\right|+\left|c^{\prime}\right|+n+1\right) \cdot\left(\left|c_{1}^{\prime \prime}\right|+\cdots+\left|c_{m}^{\prime \prime}\right|+\left|c^{\prime \prime \prime}\right|+m+1\right)$, and $|c|$ denotes the degree of $c \in C$. Diagrammatically, $\tau$ is described by:

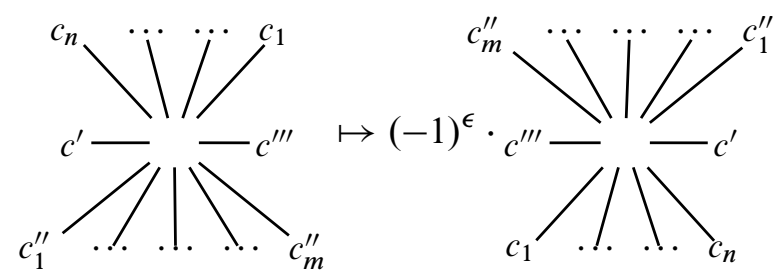


Let $(B C, D)$ be an $\mathrm{A}_{\infty}$ coalgebra. An $\mathrm{A}_{\infty}$ co-inner product

$$
F \in B_{C}^{C} C \subset \operatorname{Hom}\left(B^{C^{*}} C, B^{C} C\right)
$$

is said to be symmetric, if $F$ is invariant under $\tau$; that is,

$$
\tau(F)=F .
$$

Definition 2.3.2 An $\mathrm{A}_{\infty}$ coalgebra with $\infty$ duality consists of an $\mathrm{A}_{\infty}$ coalgebra $(B C, D)$ together with $\mathrm{A}_{\infty}$ co-inner product

$$
F:\left(B^{C^{*}} C, D^{C^{*}}\right) \rightarrow\left(B^{C} C, D^{C}\right)
$$

which is symmetric and induces an isomorphism

$$
F_{*}: H_{\bullet}\left(B^{C^{*}} C, D^{C^{*}}\right) \stackrel{\cong}{\rightarrow} H_{\bullet}\left(B^{C} C, D^{C}\right)
$$

at the level of the homology.

Let $(B C, D)$ be an $\mathrm{A}_{\infty}$ coalgebra endowed with an $\infty$ duality structure $F$ and strict counit $1 \in C$. Denote by $A=C^{*}=\operatorname{Hom}(C, R)$ the dual space of $C$ and let $C H^{\bullet}(A, M)=\prod_{n \geq 0} \operatorname{Hom}\left(A^{\otimes n}, M\right)$ be the Hochschild cochains of the $\mathrm{A}_{\infty}$ algebra $A$ with values in an $\mathrm{A}_{\infty}$ bimodule $M$. The following theorem is from [22].

Theorem 2.3.3 Let $F: B^{C^{*}} C \rightarrow B^{C} C$ denote an $\infty$ duality structure so that $F_{0,0}$ maps $C^{k}$ into $C_{d-k}$. Then, $F$ induces an isomorphism between $H H^{\bullet}\left(C^{*}, C^{*}\right)$ and $H H^{\bullet-d}\left(C^{*}, C\right)$. Moreover, transporting the cup product from $H H^{\bullet}\left(C^{*}, C^{*}\right)$ onto $H H^{\bullet}\left(C^{*}, C\right)$ and putting it together with the Connes $\triangle$ operator yields a $B V$ algebra structure.

\section{Topological constructions}

\subsection{Construction of an $\infty$ duality}

We now show the relevance of the above algebraic concepts to topology. Let $X$ be a triangulated space in which the closure of every simplex is contractible. Let $C=C \bullet X$ denote the complex of simplicial chains on $X$. We show that any closed element $\mu \in C_{\bullet} X$ gives rise to a symmetric $\mathrm{A}_{\infty}$ co-inner product $F$. Moreover, if $X$ is a Poincaré duality space and $\mu=[X]$ is its fundamental class, then $F$ is in fact an $\infty$ duality structure. We will then use this to show the Hochschild cohomology $H H^{\bullet+d}\left(C^{\bullet} X, C_{\bullet} X\right)$ is a BV algebra. We construct $F$ in a manner similar to that described by Dennis Sullivan in Appendix A, where a $\mathrm{C}_{\infty}$ structure on the rational simplicial chains is constructed. 
Definition 3.1.1 Let $\alpha$ : $\rightarrow C^{\otimes i}$ be a chain map. The map $\alpha$ is called local, if it maps a simplex $\sigma \in C$ into $C(\bar{\sigma})^{\otimes i} \subset C^{\otimes i}$, where $C(\bar{\sigma}) \subset C$ is the subcomplex of $C$ generated by the cells in the closure of $\sigma$.

Proposition 3.1.2 Let $\left(B C, D=\widetilde{D_{1}}+\widetilde{D_{2}}+\cdots\right)$ be an $A_{\infty}$ coalgebra structure on $C$ such that the components $D_{i}: C \rightarrow C^{\otimes i}$ of $D$ are local. Then, there exists a chain map $\mathcal{X}:\left(C, D_{1}\right) \rightarrow\left(B_{C}^{C} C, \mathcal{D}\right)$ of degree 0 , whose lowest component $\mathcal{X}_{0}$ is given by $D_{2}: C \rightarrow C \otimes C$.

Proof This is a proof by a double induction on the tensor degree, $n$, and the dimension of the skeleton, $r$. Thus, we will define local maps $\mathcal{X}_{j}: C \rightarrow B_{C}^{C} C_{j}$ of degree zero, $j=0,1,2, \cdots$, such that

$$
\left(\sum_{0 \leq i \leq n} \mathcal{X}_{i}\right) \circ D_{1}-\mathcal{D} \circ\left(\sum_{0 \leq i \leq n} \mathcal{X}_{i}\right)=\epsilon_{n+1}+\text { higher order terms, }
$$

where $\epsilon_{n+1}: C \rightarrow B_{C}^{C} C_{n+1}$ is a map vanishing on 0 -simplices and the higher order terms are maps $C \rightarrow \bigoplus_{i>n+1} B_{C}^{C} C_{i}$. Locality means that a simplex $\sigma \in C$ gets mapped to $B_{C(\bar{\sigma})}^{C(\bar{\sigma})} C(\bar{\sigma}) \subset B_{C}^{C} C$.

$\mathbf{n}=\mathbf{0}$ Let $\mathcal{X}_{0}=D_{2}$. This map is local by assumption and satisfies

$$
\begin{aligned}
\mathcal{X}_{0} \circ D_{1}-\mathcal{D} \circ \mathcal{X}_{0}= & D_{2} \circ D_{1} \\
& -\widetilde{D_{1}} \circ D_{2}+\left(\text { terms in } \mathcal{D} \text { higher than } D_{1}\right) \circ D_{2} \\
= & \text { higher order terms. }
\end{aligned}
$$

This is because $D_{2}$ is a chain map (compare the conditions for $D^{2}=0$ ).

$\mathbf{n} \geq \mathbf{1}$ Assume we have constructed local maps $\mathcal{X}_{j}, j=0, \cdots, n$ satisfying (3-1). We now start the induction on $r$, the dimension of the skeleton. Thus, we will construct a local $\mathcal{X}_{n+1}^{r}$, such that

$$
\begin{aligned}
\left(\sum_{0 \leq i \leq n} \mathcal{X}_{i}+\mathcal{X}_{n+1}^{r}\right) \circ D_{1}-\mathcal{D} \circ\left(\sum_{0 \leq i \leq n} \mathcal{X}_{i}+\mathcal{X}_{n+1}^{r}\right) \\
=\epsilon_{n+1}^{r}+\text { higher order terms }
\end{aligned}
$$

where $\epsilon_{n+1}^{r}: C \rightarrow B_{C}^{C} C_{n+1}$ is a map vanishing on simplices of dimension less than or equal to $r$. We may let $\mathcal{X}_{n+1}^{0}=0$, because by assumption $\epsilon_{n+1}$ vanishes on 0 -simplices.

Now, assume that a local $\mathcal{X}_{n+1}^{r}$ has been constructed. Notice that the difference $\left(\cdot \circ D_{1}\right)-(\mathcal{D} \circ \cdot): \operatorname{Hom}\left(C, B_{C}^{C} C\right) \rightarrow \operatorname{Hom}\left(C, B_{C}^{C} C\right)$ satisfies $\left(\left(\cdot \circ D_{1}\right)-(\mathcal{D} \circ \cdot)\right)^{2}=0$ 
and therefore,

$$
\begin{aligned}
0 & =\left(\left(\cdot \circ D_{1}\right)-(\mathcal{D} \circ \cdot)\right)^{2}\left(\sum_{0 \leq i \leq n} \mathcal{X}_{i}+\mathcal{X}_{n+1}^{r}\right) \\
& =\left(\left(\cdot \circ D_{1}\right)-(\mathcal{D} \circ \cdot)\right) \circ\left(\left(\cdot \circ D_{1}\right)-(\mathcal{D} \circ \cdot)\right)\left(\sum_{\substack{0 \leq i \leq n \\
\mathcal{X}_{i}}} \mathcal{X}_{n+1}^{r}\right) \\
& =\left(\left(\cdot \circ D_{1}\right)-(\mathcal{D} \circ \cdot)\right)\left(\epsilon_{n+1}^{r}+\text { higher order terms }\right) \\
& =\left[D_{1}, \epsilon_{n+1}^{r}\right]+\text { higher order terms. }
\end{aligned}
$$

Therefore, $0=\left[D_{1}, \epsilon_{n+1}^{r}\right]=\widetilde{D_{1}} \circ \epsilon_{n+1}^{r}-\epsilon_{n+1}^{r} \circ D_{1}$. Now, pick any $(r+1)-$ simplex $\sigma$. In order to construct $\mathcal{X}_{n+1}^{r+1}(\sigma)$, first notice, that $\epsilon_{n+1}^{r}$ is a local map because,

$$
\left(\epsilon_{n+1}^{r}+\text { higher order terms }\right)=\left(\left(\cdot \circ D_{1}\right)-(\mathcal{D} \circ \cdot)\right)\left(\sum_{0 \leq i \leq n} \mathcal{X}_{i}+\mathcal{X}_{n+1}^{r}\right),
$$

which is local by the locality assumptions for the $D_{i}$ 's and $\mathcal{X}_{i}$ 's, as well as the fact that the composition of two local maps is also local. Thus, for the chosen $(r+1)-$ simplex $\sigma$, the map $\epsilon_{n+1}^{r}$ restricts to a map $\left(\epsilon_{n+1}^{r}\right)^{\bar{\sigma}}: C(\bar{\sigma}) \rightarrow B_{C(\bar{\sigma})}^{C(\bar{\sigma})} C(\bar{\sigma})$, which vanishes on simplices of dimension less than $r+1$. As $\left(\epsilon_{n+1}^{r}\right)^{\bar{\sigma}}$ is a closed element in Hom $\left(C(\bar{\sigma}), B_{C(\bar{\sigma})}^{C(\bar{\sigma})} C(\bar{\sigma})\right)$ and $\bar{\sigma}$ is by assumption contractible, we have

$$
H_{\bullet}\left(\operatorname{Hom}\left(C(\bar{\sigma}), B_{C(\bar{\sigma})}^{C(\bar{\sigma})} C(\bar{\sigma})\right)\right)=\operatorname{Hom}\left(H_{0}(\bar{\sigma}), H_{\bullet}\left(B_{C(\bar{\sigma})}^{C(\bar{\sigma})} C(\bar{\sigma})\right)\right) .
$$

Since $\left(\epsilon_{n+1}^{r}\right)^{\bar{\sigma}}$ vanishes on 0 -simplices, it is not only closed, but in fact also exact.

Thus, $\left(\epsilon_{n+1}^{r}\right)^{\bar{\sigma}}$ can be written as $\left(\epsilon_{n+1}^{r}\right)^{\bar{\sigma}}=-\left[D_{1}, \rho_{n+1}^{r+1}\right]$, where $\rho_{n+1}^{r+1}: C \rightarrow B_{C}^{C} C_{n+1}$ can be assumed to be nonvanishing only on the $(r+1)$-simplex $\sigma$. We set $\mathcal{X}_{n+1}^{r+1}=$ $\mathcal{X}_{n+1}^{r}+\rho_{n+1}^{r+1}$ on $C(\bar{\sigma})$, because with this, equation (3-2) will be satisfied, because

$$
\begin{aligned}
\left(\left(\cdot \circ D_{1}\right)-(\mathcal{D} \circ \cdot)\right)\left(\bigoplus_{0 \leq i \leq n+1} \mathcal{X}_{i}+\mathcal{X}_{n+1}^{r+1}\right) \\
\quad=\left(\left(\cdot \circ D_{1}\right)-(\mathcal{D} \circ \cdot)\right)\left(\bigoplus_{0 \leq i \leq n} \mathcal{X}_{i}+\mathcal{X}_{n+1}^{r}\right)+\left(\left(\cdot \circ D_{1}\right)-(\mathcal{D} \circ \cdot)\right)\left(\mathcal{X}_{n+1}^{r+1}\right) \\
\quad=\left(\epsilon_{n+1}^{r}\right)^{\bar{\sigma}}+\text { higher order terms }+\left[D_{1}, \mathcal{X}_{n+1}^{r+1}\right]+\text { higher order terms } \\
\quad=-\left[D_{1}, \rho_{n+1}^{r+1}\right]+\left[D_{1}, \rho_{n+1}^{r+1}\right]+\text { higher order terms } \\
\quad=\text { higher order terms, }
\end{aligned}
$$

where the higher order terms now have components

$$
C(\bar{\sigma}) \rightarrow \bigoplus_{i>n+1} B_{C(\bar{\sigma})}^{C(\bar{\sigma})} C(\bar{\sigma})_{i}
$$


By the construction, it follows that $\mathcal{X}_{n+1}^{r+1}$ coincides on the boundary of two $(r+1)-$ simplices, and therefore gives rise a local map $\mathcal{X}_{n+1}^{r+1}$.

Finally, notice, that $\epsilon_{n+2}$ vanishes on 0 -simplices, since the left-hand side of (3-2) vanishes on 0 -simplices for any $r$. This completes the induction and the proof of the proposition.

Proposition 3.1.3 Let $X$ be a compact and triangulated space in which the closure of every simplex is contractible. Let $(B C, D)$ be a local $A_{\infty}$ coalgebra structure, where $D_{1}$ and $D_{2}$ are the boundary operator and comultiplication on the on $C=C_{\bullet} X$, respectively. For every closed cycle $\mu \in C$, there exists a symmetric $A_{\infty}$ co-inner product $F \in B_{C}^{C} C$ such that the lowest component of $F$ is given by $\frac{1}{2}\left(D_{2}(\mu)+\tau\left(D_{2}(\mu)\right)\right) \in C \otimes C=B_{C}^{C} C_{0}$, where $\tau$ is the rotation map from equation (2-4).

Proof Using Proposition 3.1.2, we obtain a chain map $\mathcal{X}:\left(C, D_{1}\right) \rightarrow\left(B_{C}^{C} C, \mathcal{D}\right)$. Let us define an $\mathrm{A}_{\infty}$ co-inner product by setting $F^{0}=\mathcal{X}(\mu) \in B_{C}^{C} C . F^{0}$ is in fact an $\mathrm{A}_{\infty}$ co-inner product, since it is closed under $\mathcal{D}, \mathcal{D}\left(F^{0}\right)=\mathcal{D}(\mathcal{X}(\mu))=\mathcal{X}\left(D_{1}(\mu)\right)=$ $\mathcal{X}(0)=0$. Now, let $F=\left(F^{0}+\tau\left(F^{0}\right)\right) / 2$. It is clear that $\tau(F)=F$, since $\tau^{2}=$ id, and that $\mathcal{D}(F)=0$, since $\tau \mathcal{D}= \pm \mathcal{D} \tau$.

Theorem 3.1.4 Let $X$ be a compact and triangulated Poincaré duality space in which the closure of every simplex is contractible and let $\mu \in C_{d} X$ denote its fundamental class. Then, the resulting $F \in B_{C}^{C} C \subset \operatorname{Hom}\left(B^{C^{*}} C, B^{C} C\right)$ is an $\infty$ duality structure. That is to say $F$ is a co-inner product which is symmetric and induces an isomorphism

$$
F_{*}: H_{\bullet}\left(B^{C^{*}} C, D^{C^{*}}\right) \stackrel{\cong}{\rightarrow} H_{\bullet}\left(B^{C} C, D^{C}\right),
$$

at the level of the homology.

Proof Choose a strictly cocommutative simplicial chain model $C=C_{\bullet} X$ for $X$. This can always be achieved by symmetrization of a given coproduct. Complete this model to a local $\mathrm{A}_{\infty}$ coalgebra structure on $C$ as described in Appendix A by Dennis Sullivan.

Recall that in Proposition 3.1.3 the lowest component $F_{0,0}: C^{*} \rightarrow C$ of the $\mathrm{A}_{\infty}$ co-inner product is given by capping a cochain with the fundamental cycle $\mu$, because for $a, b \in C^{*}$, it is $(a \otimes b)\left(D_{2}(\mu)\right)=(a \cap \mu)(b)$, where $D_{2}$ was chosen to be cocommutative. Since $X$ satisfies Poincaré duality, it follows that $F_{0,0}: C^{*} \rightarrow C$ induces an isomorphism on homology. Thus, Theorem 2.2.5 implies the claim. 
Remark 3.1.5 Let us say a few words about a related and important issue. Although our construction of the $\mathrm{A}_{\infty}$ co-inner product gives rise to a locally defined structure, the quasi-inverse, which we show to exist using minimal models, is not necessarily local. This is simply on account of the fact that the lowest level part of the inverse map is given by a representative for the Thom class of the diagonal $X \hookrightarrow X \times X$ and that a result of Mc Crory [16] states the existence of such a local Thom class implies the Poincare duality space is a homology manifold (In fact, if the dimension of $X$ is greater than 4, a result of Galewski and Stern [6] implies that $X$ has the homotopy type of a topological manifold.) So, as Sullivan pointed out to us, an $\infty$ duality structure with a local quasi-inverse should give rise to an $X$-controlled Poincaré complex a la Ranicki as well as to an L-theory orientation $[X]_{\mathbb{L}} \in H_{n}(X, \mathbb{L})$ for $X$. Here, $\mathbb{L}$ denotes the L-theory spectrum. For an account of algebraic L-theory and how to obtain rational Pontrjagin classes, see Ranicki [18].

\subsection{BV structure on $\mathrm{HH}^{\bullet+\mathrm{d}}\left(\mathrm{C}^{\bullet} \mathrm{X}, \mathrm{C} \bullet \mathrm{X}\right)$}

In this section, $X$ is a compact and triangulated space Poincare duality space of dimension $d$, and $\mu \in C=C_{d} X$ represents its fundamental class. We use the $\infty$ duality structure of Proposition 3.1.3 to obtain a BV algebra on the Hochschild complex $H H^{\bullet+d}\left(C^{\bullet} X, C_{\bullet} X\right)$. For this, we use a result from [22], namely, that the Hochschild cohomology of an $\mathrm{A}_{\infty}$ coalgebra with $\infty$ duality is a $\mathrm{BV}$ algebra with a unit.

Corollary 3.2.1 Let $X$ be a compact and triangulated Poincaré duality space in which the closure of every simplex is contractible. Then, the shifted Hochschild cohomology $H H^{\bullet+d}\left(C^{\bullet} X, C \bullet X\right)$ is a $B V$ structure with a unit.

Proof This follows immediately by applying the $\infty$ duality structure obtained in Theorem 3.1.4 to Theorem 2.3.3.

Jones proved in [8] that for a simply connected space $X$ the homology of the free loop space $H_{\bullet} L X$ is identified with the Hochschild cohomology $H H^{\bullet}\left(C^{\bullet} X, C \bullet X\right)$. One implication is as follows,

Corollary 3.2.2 Let $X$ be a compact, triangulated, and simply connected Poincaré duality space. Then, the shifted homology of the free loop space, $H_{\bullet+d} L X$, is a $B V$ algebra with a unit.

This corollary should be viewed in light of the seminal work of Chas and Sullivan. They showed that there exists a natural BV structure on $H_{\bullet+d} L X$, when $X$ is a 
closed manifold [1]. It is therefore natural to ask whether, for a simply connected and closed smooth manifold, the BV structure in Corollary 3.2.2 coincides with that defined in [1]. In fact, developing an algebraic model for the Chas-Sullivan string topology [1] was one of the original motivations for this work. The identification $H_{\bullet} L X \cong H H^{\bullet}\left(C^{\bullet} X, C \bullet X\right)$, due to Jones [8], is explicit. One can see that the $\Delta$ operator used in string topology coincides with the $\Delta$ operator on $H H^{\bullet}\left(C^{\bullet} X, C \bullet X\right)$, used in Corollary 3.2.1. One can ask whether the product presented here also coincides with the Chas-Sullivan product. Although there is some evidence that this is indeed the case, the question remains open. Also, there are identification of the products in the literature (see Cohen and Jones [4], Merkulov [17] and Felix, Thomas and Vigue-Poirrier [26]), but it is not a priori clear that they are the same as the one coming from the $\mathrm{A}_{\infty}$ Poincaré duality structure.

\section{Appendix A Local construction of $\infty$ structures}

\section{DENNIS SULLIVAN}

Let $X$ be a cell complex with cells $e_{\alpha}$ so that the closure $\bar{e}_{\alpha}\left(=e_{\alpha}\right.$ union faces of $e_{\alpha}$ ) have the homology of a point ( $\mathbb{Q}$-coefficients). Let $L(X)=L$ denote the free Lie algebra with generators $e_{\alpha}$ placed in degrees (dimension $e_{\alpha}$ ) -1 . Consider $L$ as a direct sum $L_{0} \oplus L_{1} \oplus L_{2} \oplus \cdots$, where $L_{0}$ is the ground field $\mathbb{Q}, L_{1}$ is the linear span of the generators $e_{\alpha}, L_{2}$ is spanned by brackets of pairs of elements in $L_{1}$, etc. Consider derivations $\delta$ of $L$ expanded into components $\delta=\delta_{0}+\delta_{1}+\delta_{2}+\cdots$ where $\delta_{k}$ is determined by a linear mapping $L_{1} \rightarrow L_{k}$ of degree -1 . Here we discuss only the special case where $\delta_{0}=0$.

Orient the cells of $X$ and let $\delta_{1}$ be the boundary operator (shifted down by 1) $L_{1} \rightarrow L_{1}$. Note $\delta_{1}$ still has degree -1 . Choose a local canonical cellular approximation to the diagonal mapping (as in the proof below) to obtain $L_{1} \rightarrow L_{2}$. Extend $\delta_{1}$ and $\delta_{2}$ to derivations of $L$ and also call them $\delta_{1}$ and $\delta_{2}$.

Theorem A.1 There is a canonical local inductive construction of

$$
L_{1} \stackrel{\delta_{3}}{\rightarrow} L_{3}, L_{1} \stackrel{\delta_{4}}{\rightarrow} L_{4}, \cdots
$$

so the total derivation $\delta=\delta_{1}+\delta_{2}+\cdots$ satisfies $\delta \circ \delta=0$.

Remark A.2 By local we mean $\delta e_{\alpha}$ belongs in the sub Lie algebra generated by the cells in the closure of $e_{\alpha}$. 
Proof We interpret $\delta \circ \delta=0$ as the equation $[\delta, \delta]=0$, where $[\cdot, \cdot]$ is the graded commutator. For any $\delta$ the Jacobi identity is $[\delta,[\delta, \delta]]=0$. Suppose $\delta^{k}=\delta_{1}+\cdots+\delta_{k}$ has been defined so that $\left[\delta^{k}, \delta^{k}\right]$ has the first nonzero term in monomial degree $k+1$. Jacobi implies this error commutes with $\delta_{1}$; that is, it is a closed element in the complex $\operatorname{Der}(L)$ of derivations of $L$. If we work in the closure of a cell, the homology hypothesis implies that $\operatorname{Der}(L)$ has homology only in degrees 0 and -1 . Therefore, the error, which lives in degree -2 , can be written as a commutator with $\delta_{1}$. Using the cells to generate a linear basis of each $L_{k}$ by bracketing, we choose this solution to lie in the image of the adjoint of $\delta_{1}$ to make it canonical. This canonical solution is $\delta_{k+1}$ and this completes the induction, since one knows at the beginning $\delta_{1} \circ \delta_{1}=0$ and $\delta_{2}$ is chain mapping; that is, $\left[\delta_{2}, \delta_{1}\right]=0$.

Remark A.3 (1) The canonical $\delta$ respects any cellular symmetry of the cell.

(2) For the zero cell $a$, then $\delta a+1 / 2[a, a]=0$, if we choose the diagonal approximation to send a vertex $a$ to $-a \otimes a$.

(3) For the one cell $e$ with vertices $a, b$, choose an orientation so that $\delta_{1} e=b-a$ and let $\operatorname{ad}_{e}=[e, \cdot]$. On the one hand, the procedure of this appendix gives a differential of the form

$$
\delta e=\sum_{i=0}^{\infty} \alpha_{i}\left(\operatorname{ad}_{e}\right)^{i}(a)+\beta_{i}\left(\operatorname{ad}_{e}\right)^{i}(b),
$$

and on the other hand, we gave the following specific infinity structure for the interval in [12]:

$$
\delta e=\left(\operatorname{ad}_{e}\right) b+\sum_{i=0}^{\infty} \frac{B_{i}}{i !}\left(\operatorname{ad}_{e}\right)^{i}(b-a),
$$

where $B_{i}$ denotes the $i$-th Bernoulli number. These two formulae are conjecturally the same.

(4) The higher terms $\delta_{4}, \delta_{5}, \cdots$ have the interpretation of the rest of the higher homotopies in the structure of an infinity homotopy cocommutative and coassociative coalgebra structure on the chains of $X$; that is, a $C_{\infty}$ structure.

(5) The argument used here can be employed in a variety of contexts to construct local $\infty$-structures. For instance, let $C_{\bullet} X$ denote the complex of chains on $X$ and $T C_{\bullet} X$ the tensor algebra generated by $C_{\bullet} X$. Similarly, for a cell $e_{\alpha}, C_{\bullet} \bar{e}_{\alpha}$ denotes the chain complex of the closure of $e_{\alpha}$ and $T C_{\bullet} \bar{e}_{\alpha}$ its tensor algebra. Working in the closure of a cell, $T C_{\bullet} \bar{e}_{\alpha}$ has homology $\mathbb{Q}$ in degrees $-1,-2,-3, \cdots$ and therefore $\operatorname{Der}\left(T C_{\bullet} \bar{e}_{\alpha}\right)$ has homology $\mathbb{Q}$ in degrees $0,-1,-2, \cdots$. The error is a class in the homology group $H_{\bullet}\left(\operatorname{Der}\left(T C_{\bullet} \bar{e}_{\alpha}\right)\right)=\operatorname{Hom}\left(H_{0}\left(\bar{e}_{\alpha}\right), H_{\bullet}\left(T C_{\bullet} \bar{e}_{\alpha}\right)\right)$. Let us call an 
element $\epsilon \in \operatorname{Hom}\left(C_{\bullet} X, T C_{\bullet} X\right)$ local, if for any cell $\bar{e}_{\alpha} \subset X, \epsilon\left(C_{\bullet} \bar{e}_{\alpha}\right) \subset T C_{\bullet}\left(\bar{e}_{\alpha}\right)$. We perform a double induction for both the tensor degree $k$ and the cell degree $n$. Assume $\delta^{k-1}=\delta_{1}+\cdots+\delta_{k-1}$ is defined as a local map on the $n$-skeleton, such that $\left[\delta^{k-1}, \delta^{k-1}\right]=0$ up to tensor degree $k$. Furthermore, assume that $\delta^{k-1}$ is already extended on the $(n-1)$-skeleton to a local map $\delta^{k}=\delta_{1}+\cdots+\delta_{k-1}+\delta_{k}$, such that $\left[\delta_{k}, \delta_{k}\right]=0$ up to tensor degree $k+1$. We will show that $\delta^{k}$ can be extended to the $n$-skeleton, such that $\delta^{k}$ coincides with the previous map on the faces (ie locality), and $\left[\delta^{k}, \delta^{k}\right]=0$ up to tensor degree $k+1$. To this end, look at $\epsilon=\left[\delta^{k}, \delta^{k}\right]$ on an $n$-cell $e_{\alpha} . \epsilon$ has a lowest tensor degree of $k$, and vanishes on the faces of $e_{\alpha}$. Jacobi identity shows, that $\epsilon$ is closed under the differential given by the commutator with $\delta_{1}$. The complex $\left.\operatorname{Hom}\left(C_{\bullet} \bar{e}_{\alpha}, T C_{\bullet} \bar{e}_{\alpha}\right)\right)$ has homology $H_{\bullet}\left(\operatorname{Hom}\left(C_{\bullet} \bar{e}_{\alpha}, T C_{\bullet} \bar{e}_{\alpha}\right)\right)=$ $\operatorname{Hom}\left(H_{0}\left(\bar{e}_{\alpha}\right), H_{\bullet}\left(T C_{\bullet} \bar{e}_{\alpha}\right)\right)$. Now, $\epsilon$ is closed and vanishes on 0 -cells, so it represents the zero homology class; that is, it must be exact. Let $\epsilon=\left[\delta_{1}, \rho\right]$. Since $\epsilon$ vanishes on all faces of $e_{\alpha}$, we may set $\rho$ equal to zero on all $m$-cells, for all $m \leq n-1$, and still have the relation $\epsilon=\left[\delta_{1}, \rho\right]$. Define $\delta_{k}$ to be the old $\delta_{k}$ plus $\rho$. Then, $\left[\delta^{k}, \delta^{k}\right]=0$ up to tensor degree $k+1$ on all of $e_{\alpha}$, and $\delta^{k}$ is local. To start the induction, choose $\delta_{1}$ to be the boundary operator and $\delta_{2}$ be a local comultiplication, which is both cocommutative and coassociative on zero cells. Define $\delta$ on a $0-$ cell by

$$
\delta_{k}=\left\{\begin{array}{c}
\delta_{k}, \text { for } k=1,2 \\
0, \text { for } k \geq 3 .
\end{array}\right.
$$

Thus, one extends, by induction on both $n$ and $k$, a local map $\delta$ to the all cells in the $n$-skeleton, such that $[\delta, \delta]=0$.

\section{Appendix B The one-dimensional case}

In this section we treat the one-dimensional case of the circle. To this end, we calculate the $\mathcal{X}$ map from Proposition 3.1.2 for a point, the unit interval, and then for the circle. We will see that even in this simplest of cases, the higher homotopies can not be avoided.

\section{B.1 Compatibility relations for $\mathcal{X}$}

Let us start by describing the compatibility relation for extending the $\mathcal{X}$ map to the $n$-skeleton once it has been defined on the $(n-1)$-skeleton of a closed, triangulated topological space $X$. Using Proposition 3.1.2, one can construct a map $\mathcal{X}:\left(C, D_{1}\right) \rightarrow$ $\left(B_{C}^{C} C, \mathcal{D}\right)$, where $C=C_{\bullet} X$ is a simplicial model of $X$ in which the closure of simplices are contractible. We will write $A$ for the cochain model $A=C^{\bullet} X$. The goal is to build an explicit map $\mathcal{X}$ inductively over the $n$-skeletons of $C$. We will 
sometimes ignore the grading and simply write $A$ instead of the shifted $s A$, keeping in mind that the correct grading is always given after shifting.

In detail, $\mathcal{X}: C \rightarrow B_{C}^{C} C$ being a chain map means that for all $\sigma \in C$, one has $\mathcal{X}\left(D_{1}(\sigma)\right)=\mathcal{D}(\mathcal{X}(\sigma)) \in B_{C}^{C} C \subset \operatorname{Hom}\left(T_{A}^{A} A, R\right)$. Since $\mathcal{X}(\sigma) \in \operatorname{Hom}\left(T_{A}^{A} A, R\right)$ denotes a sequence of inner products, we will use the shorthand

$$
\langle\cdots\rangle^{\sigma}=\mathcal{X}(\sigma): T_{A}^{A} A \rightarrow R,
$$

written in components as $\langle\cdots\rangle_{k, l}^{\sigma}: A^{\otimes k} \otimes A \otimes A^{\otimes l} \otimes A \rightarrow R$. (The arguments from $A$ are being applied into the dots.) Then, $\mathcal{X}\left(D_{1}(\sigma)\right)=\mathcal{D}(\mathcal{X}(\sigma))$ reads

$$
\langle\cdots\rangle^{D_{1}(\sigma)}=\left\langle\mathcal{D}^{*}(\cdots)\right\rangle^{\sigma} .
$$

Below, we will use the Alexander-Whitney comultiplication $\Delta: C \rightarrow C \otimes C$,

$$
\Delta\left(v_{0}, \cdots, v_{n}\right)=\sum_{i=1}^{n}\left(v_{0}, \cdots, v_{i}\right) \otimes\left(v_{i}, \cdots, v_{n}\right),
$$

which makes $C$ into a strictly coassociative differential graded coalgebra. In the strictly coassociative case, the differential $\mathcal{D}$ only has two components, namely the differential $D_{1}$ and the comultiplication $D_{2}$, because with this $D=\widetilde{D_{1}}+\widetilde{D_{2}}$ satisfies $D^{2}=0$. Thus, the chain map condition for $\mathcal{X}$ becomes

$$
\langle\cdots\rangle^{D_{1}(\sigma)}=\left\langle{\widetilde{D_{1}^{*}}}^{*}(\cdots)\right\rangle^{\sigma}+\left\langle{\widetilde{D_{2}^{*}}}^{*}(\cdots)\right\rangle^{\sigma} .
$$

We will use the notation $\delta(a)=D_{1}^{*}(a)$ and $a \cdot b=D_{2}^{*}(a, b)$. The symbol $\widetilde{D_{1}^{*}}$ denotes the extension of $D_{1}^{*}$ to a derivation on $T_{A}^{A} A$, and similarly $\widetilde{D_{2}^{*}}$ denotes the extension of $D_{2}^{*}$ to a derivation on $T_{A}^{A} A$. It is important to note that two special elements never combine [23]. For example, if we underline the two special elements, then we have the maps

$$
\begin{aligned}
& \widetilde{D_{1}^{*}}(a, \underline{b}, c, \underline{d})=(\delta(a), \underline{b}, c, \underline{d})+(-1)^{\|a\|}(a, \delta(b), c, \underline{d}) \\
& +(-1)^{\|a\|+\|b\|}(a, \underline{b}, \delta(c), \underline{d})+(-1)^{\|a\|+\|b\|+\|c\|}(a, \underline{b}, c, \underline{\delta(d)}) \\
& \widetilde{D_{2}^{*}}(a, \underline{b}, c, \underline{d})=(\underline{a \cdot b}, c, \underline{d})+(-1)^{\|a\|}(a, \underline{b \cdot c}, \underline{d}) \\
& +(-1)^{\|a\|+\|b\|}(a, \underline{b}, \underline{c \cdot d})+(-1)^{\epsilon}(\underline{b}, c, \underline{d \cdot a}) \\
& \widetilde{D_{2}^{*}}(a, b, \underline{c}, \underline{d})=(a \cdot b, \underline{c}, \underline{d})+(-1)^{\|a\|}(a, \underline{b \cdot c}, \underline{d}) \\
& +(-1)^{\epsilon}(b, \underline{c}, \underline{d \cdot a}) \\
& \widetilde{D_{2}^{*}}(\underline{a}, b, c, \underline{d})=(\underline{a \cdot b}, c, \underline{d})+(-1)^{\|a\|}(\underline{a}, b \cdot c, \underline{d}) \\
& +(-1)^{\|a\|+\|b\|}(\underline{a}, b, \underline{c \cdot d}) \text {, }
\end{aligned}
$$

where $\epsilon=\|b\|+\|c\|+\|a\| \cdot(\|b\|+\|c\|+\|d\|)$, and $\|x\|$ denotes the degree in $s A$. 
Equation (B-1) shows that it is possible to define $\langle\cdots\rangle^{\sigma}$ inductively on the skeleton, because one only needs to know the lower components $\langle\cdots\rangle^{D_{1}(\sigma)}$. Let ${ }^{n} \mathcal{X}$ denote the $\mathcal{X}$ map for the standard $n$-simplex $\Delta^{n}$. We will define ${ }^{n} \mathcal{X}: C_{\bullet} \Delta^{n} \rightarrow B_{C_{\bullet} \Delta^{n}} C_{\bullet} \Delta^{n}$ inductively by extending the map ${ }^{n-1} \mathcal{X}$ of the standard $(n-1)-\operatorname{simplex} \Delta^{n-1}$. If one identifies $\Delta^{n-1}$ with a face of $\Delta^{n}$, then ${ }^{n} \mathcal{X}$ is defined on this face of $\Delta^{n}$ by requiring the commutativity of the following diagram:

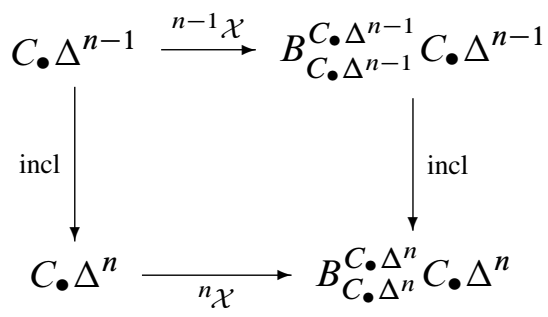

It follows, that for a given triangulated topological space $X$ with simplicial chain model $C=C_{\bullet} X$, one can use the ${ }^{n} \mathcal{X}$ to define $\mathcal{X}: C \rightarrow B_{C}^{C} C$. Namely, for an $n$-simplex $\sigma$ of $X$, which is identified with the standard $n$-simplex $\Delta^{n}$, one requires the following diagram to commute:

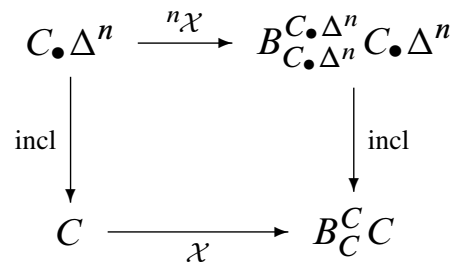

\section{B.2 The 0-simplex}

In this section, we calculate the map $\mathcal{X}$ for the trivial case $\Delta^{0}=\{a\}$. Let $C=C_{\bullet}\{a\}$ be of the 0 -simplex, and $s A=s C^{\bullet}\{a\}$ be the shifted cochain model, generated by one element $a$ in degree 1 .

Proposition B.1 If the differential graded algebra structure is given by $\delta(a)=0$ and $a \cdot a=a$, then $\mathcal{X}(a)=a \otimes a \in C \otimes C$ defines an $A_{\infty}$ inner product. More precisely, $\mathcal{X}(a)$ is given by the inner products

$$
\begin{aligned}
\langle a, a\rangle_{0,0}^{a} & =1, \\
\langle a, \cdots, a\rangle_{i, j}^{a} & =0, \quad \text { for } i+j>0 .
\end{aligned}
$$

Proof We show that the inner products satisfy equation (B-1). The first two terms $\langle\cdots\rangle^{D_{1}(a)}$ and $\left\langle{\widetilde{D_{1}^{*}}}^{*}(\cdots)\right\rangle^{a}$ always vanish, because $D_{1}(a)=0 \in C$ and $\delta(a)=0 \in A$. 
We need to show that $\left\langle\widetilde{D}_{2}^{*}(\cdots)\right\rangle^{a}=0$. In order to check this, we examine $\left\langle\widetilde{D_{2}^{*}}(\cdots)\right\rangle^{a}$ by inserting elements from $T_{A}^{A} A$. Notice that the multiplication takes exactly two inputs and $\widetilde{D_{2}^{*}}$ decreases the monomial degree of an element in $T_{A}^{A} A$ by one, and that the inner product $\mathcal{X}(a)=\langle\cdots\rangle^{a}$ is nonzero only in the component $A \otimes A=T_{A}^{A} A_{0}=$ $A^{\otimes 0} \otimes A \otimes A^{\otimes 0} \otimes A$. Therefore, we need to test equation (B-1) only for elements of $T_{A}^{A} A_{1}=\left(A^{\otimes 1} \otimes A \otimes A^{\otimes 0} \otimes A\right) \oplus\left(A^{\otimes 0} \otimes A \otimes A^{\otimes 1} \otimes A\right):$

$$
\begin{aligned}
& \left\langle\widetilde{D_{2}^{*}}(a, a, a)\right\rangle_{1,0}^{a}=\langle a \cdot a, a\rangle_{0,0}^{a}-\langle a, a \cdot a\rangle_{0,0}^{a}=1-1=0 \\
& \left\langle\widetilde{D_{2}^{*}}(a, a, a)\right\rangle_{0,1}^{a}=\langle a \cdot a, a\rangle_{0,0}^{a}-\langle a, a \cdot a\rangle_{0,0}^{a}=1-1=0
\end{aligned}
$$

\section{B.3 The 1-simplex}

Next, we calculate $\mathcal{X}$ for the case of the interval. Let $\Delta^{1}=I=[a, b]$ be an interval, oriented from $a$ to $b$.

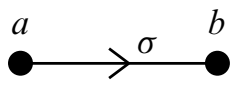

Denote by $C=C_{\bullet} I$ the chain model for the interval given by the generators $a, b$ and $\sigma$. Furthermore, let $s A=s^{\bullet} I$ be the shifted cochain model, which, by slight abuse of notation, has generators $a$ and $b$ in degree 1 , and $\sigma$ in degree 0 . The differential graded algebra structure of $s A$ is given by

$$
\begin{gathered}
\delta(a)=\sigma, \quad \delta(b)=-\sigma, \quad \delta(\sigma)=0, \\
a \cdot a=a, \quad b \cdot b=b, \\
\sigma \cdot a=-\sigma, \quad b \cdot \sigma=\sigma,
\end{gathered}
$$

and

and all other multiplications vanish. Note that after the shift the associativity implies $D_{2}^{*}\left(D_{2}^{*}(x, y), z\right)=(-1)^{\|x\|} D_{2}^{*}\left(x, D_{2}^{*}(y, z)\right)$, where $\|x\|$ denotes the degree in $s A$. Notice that $s A$ is highly noncommutative, eg $\sigma \cdot a=-\sigma$, but $a \cdot \sigma=0$.)

Proposition B.2 Let $\mathcal{X}(a)=a \otimes a$ and $\mathcal{X}(b)=b \otimes b$; that is,

$$
\begin{aligned}
& \langle a, a\rangle_{0,0}^{a}=1, \\
& \langle b, b\rangle_{0,0}^{b}=1,
\end{aligned}
$$

and define $\mathcal{X}(\sigma)$ by the following sequence of inner products on $s A$ :

$$
\begin{array}{ll}
\langle\sigma, \cdots, \sigma, a\rangle_{k, 0}^{\sigma}=1 & k \geq 0 \\
\langle\sigma, \cdots, \sigma, b, \sigma\rangle_{k, 0}^{\sigma}=-1 & k \geq 0,
\end{array}
$$


and $\mathcal{X}$ is zero otherwise. Then, $\mathcal{X}: C \rightarrow B_{C}^{C} C$ is a chain map, where $C=C_{\bullet} I$.

The proof of the above proposition is a case by case calculation which, in spite of its length, is straightforward and therefore omitted.

The inner products of $\mathcal{X}(\sigma)$ can be expressed diagrammatically [23]. For example for $k=5$, we have:
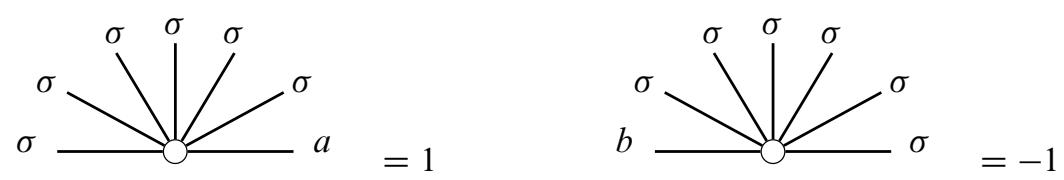

Remark B.3 The lowest component of $\mathcal{X}$ is given by $D_{2}$. Therefore, the inner products $\langle b, \sigma\rangle^{\sigma}$ and $\langle\sigma, a\rangle^{\sigma}$ are clearly nonzero; see Proposition 3.1.2. Let us examine some of the higher inner products, for example $(b, \underline{b}, \underline{\sigma})$, with special elements underlined. Equation (B-1) says:

$$
\begin{aligned}
\langle b, \underline{b}, \underline{\sigma}\rangle_{1,0}^{D_{1}(\sigma)} & =\langle b, \underline{b}, \underline{\sigma}\rangle_{1,0}^{a}-\langle b, \underline{b}, \underline{\sigma}\rangle_{1,0}^{b}=0 \\
\left\langle\widetilde{D}_{1}^{*}(b, \underline{b}, \underline{\sigma})\right\rangle_{1,0}^{\sigma} & =\langle\sigma, \underline{b}, \underline{\sigma}\rangle_{1,0}^{\sigma}-\langle b, \underline{\sigma}, \underline{\sigma}\rangle_{1,0}^{\sigma} \\
\left\langle\widetilde{D}_{2}^{*}(b, \underline{b}, \underline{\sigma})\right\rangle_{1,0}^{\sigma} & =\langle\underline{b} \cdot \underline{b}, \underline{\sigma}\rangle_{0,0}^{\sigma}=\langle\underline{b}, \underline{\sigma}\rangle_{0,0}^{\sigma} \neq 0
\end{aligned}
$$

This shows that at least one of the two inner products $\langle\sigma, \underline{b}, \underline{\sigma}\rangle^{\sigma}$ or $\langle b, \underline{\sigma}, \underline{\sigma}\rangle^{\sigma}$ have to be nonzero in order for equation $(\mathrm{B}-1)$ to be satisfied. This example also shows that there is a choice, which inner products to put nonzero. We chose $\langle\sigma, \underline{b}, \underline{\sigma}\rangle^{\sigma} \neq 0$, but one could also choose $\langle b, \underline{\sigma}, \underline{\sigma}\rangle^{\sigma} \neq 0$. This choice would result in another possible $\mathrm{A}_{\infty}$ co-inner product. It turns out that the choice from Proposition B.2 gives the $\mathrm{A}_{\infty}$ co-inner product which has the least amount of nonzero components.

After having calculated the map $\mathcal{X}$ for the two lowest dimensional simplices $\Delta^{0}$ and $\Delta^{1}$, it is desirable to extend it to higher-dimensional simplices. Unfortunately, an explicit calculation of $\mathcal{X}$ for higher simplices becomes considerably more complicated than the cases considered above.

\section{B.4 The circle}

We can now use Proposition B.2 to find an $\mathrm{A}_{\infty}$ co-inner product on a model for the circle given by the following simplicial complex: 


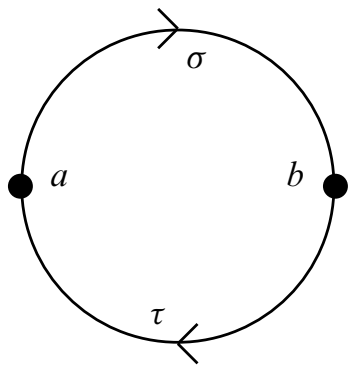

Let $C=C_{\bullet} S^{1}$, and $s A=s C^{\bullet} S^{1}$ be the shifted cochain model of the circle with generators $a, b, \sigma$ and $\tau$, as indicated above. The differential is given by

$$
\begin{array}{ll}
\delta(a)=\sigma-\tau, & \delta(b)=\tau-\sigma, \\
\delta(\sigma)=0, & \delta(\tau)=0,
\end{array}
$$

and the multiplication is nonzero only on the generators

$$
\begin{array}{lll}
a \cdot a=a, & \sigma \cdot a=-\sigma, & a \cdot \tau=\tau, \\
b \cdot b=b, & b \cdot \sigma=\sigma, & \tau \cdot b=-\tau .
\end{array}
$$

Again we abuse notation by using the same notation for an element in $C$ and its dual $A$. The fundamental cycle of $C$ is $\mu=\sigma+\tau \in C$. Then, by Proposition 3.1.3, we know that an $\mathrm{A}_{\infty}$ co-inner product is given by $\mathcal{X}(\mu)=\mathcal{X}(\sigma)+\mathcal{X}(\tau)$. Namely, the only nonzero components of the $\mathrm{A}_{\infty}$ co-inner product are as follows. For all $k \geq 0$, we have

$$
\begin{aligned}
\langle\sigma, \cdots, \sigma, a\rangle_{k, 0} & =1, \\
\langle\tau, \cdots, \tau, a, \tau\rangle_{k, 0} & =-1, \\
\langle\sigma, \cdots, \sigma, b, \sigma\rangle_{k, 0} & =-1, \\
\langle\tau, \cdots, \tau, b\rangle_{k, 0} & =1 .
\end{aligned}
$$

The following diagrams exhibit the case for $k=5$ :
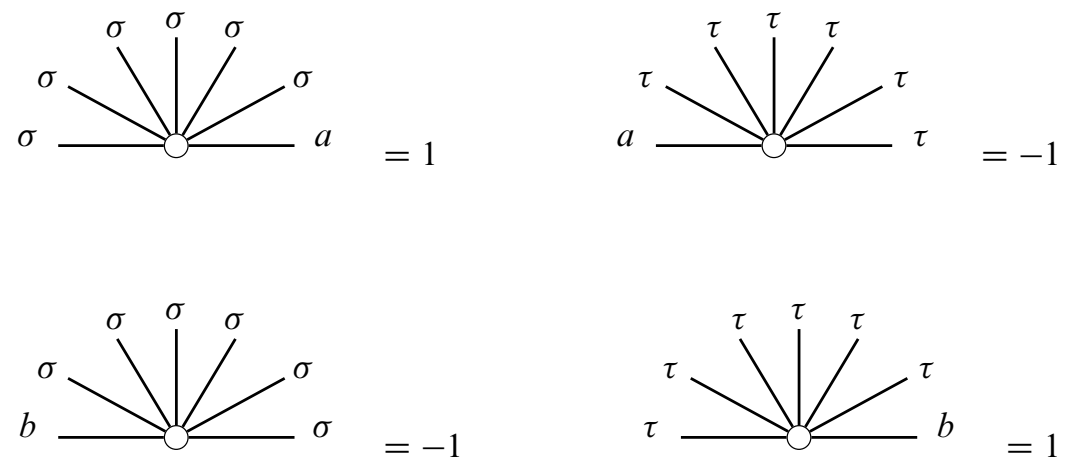


\section{References}

[1] M Chas, D Sullivan, String topology arXiv:math.GT/9911159

[2] M Chas, D Sullivan, Closed string operators in topology leading to Lie bialgebras and higher string algebra, from: "The legacy of Niels Henrik Abel”, Springer, Berlin (2004) 771-784 MR2077595

[3] D Chataur, A bordism approach to string topology, Int. Math. Res. Not. (2005) 28292875 MR2180465

[4] R L Cohen, J D S Jones, A homotopy theoretic realization of string topology, Math. Ann. 324 (2002) 773-798 MR1942249

[5] R L Cohen, J Klein, D Sullivan, The homotopy invariance of the string topology loop product and string bracket arXiv:math.GT/0509667

[6] D E Galewski, R J Stern, The relationship between homology and topological manifolds via homology transversality, Invent. Math. 39 (1977) 277-292 MR0445507

[7] A Gómez-Tato, S Halperin, D Tanré, Rational homotopy theory for non-simply connected spaces, Trans. Amer. Math. Soc. 352 (2000) 1493-1525 MR1653355

[8] J D S Jones, Cyclic homology and equivariant homology, Invent. Math. 87 (1987) 403-423 MR870737

[9] H Kajiura, Noncommutative homotopy algebras associated with open strings arXiv: math.QA/0306332

[10] J R Klein, Fiber products, Poincaré duality and $A_{\infty}$-ring spectra, Proc. Amer. Math. Soc. 134 (2006) 1825-1833 MR2207500

[11] M Kontsevich, Deformation quantization of Poisson manifolds, Lett. Math. Phys. 66 (2003) 157-216 MR2062626

[12] R Lawrence, D Sullivan, A free differential Lie algebra for the interval arXiv: math. AT/0610949 v2 31 Oct 2006

[13] R Longoni, T Tradler, Infinity inner products for cyclic operads arXiv: math.AT/0312231

[14] M Markl, A cohomology theory for A(m)-algebras and applications, J. Pure Appl. Algebra 83 (1992) 141-175 MR1191090

[15] M Markl, Homotopy algebras are homotopy algebras, Forum Math. 16 (2004) 129-160 MR2034546

[16] C McCrory, A characterization of homology manifolds, J. London Math. Soc. (2) 16 (1977) 149-159 MR0445506

[17] S A Merkulov, De Rham model for string topology, Int. Math. Res. Not. (2004) 29552981 MR2099178 
[18] A A Ranicki, Algebraic L-theory and topological manifolds, Cambridge Tracts in Mathematics 102, Cambridge University Press, Cambridge (1992) MR1211640

[19] J D Stasheff, Homotopy associativity of $H$-spaces. I, II, Trans. Amer. Math. Soc. 108 (1963), 275-292; ibid. 108 (1963) 293-312 MR0158400

[20] D Sullivan, Infinitesimal computations in topology, Inst. Hautes Études Sci. Publ. Math. (1977) 269-331 (1978) MR0646078

[21] D Sullivan, Open and closed string field theory interpreted in classical algebraic topology, from: "Topology, geometry and quantum field theory", London Math. Soc. Lecture Note Ser. 308, Cambridge Univ. Press, Cambridge (2004) 344-357 MR2079379

[22] T Tradler, The BV algebra on Hochschild cohomology induced by infinity inner products arXiv:math.QA/0210150

[23] T Tradler, $\infty$-inner products on $A_{\infty}$-Algebras arXiv:math. AT/0108027

[24] T Tradler, M Zeinalian, Algebraic string operations arXiv:math.QA/0605770

[25] T Tradler, M Zeinalian, On the cyclic Deligne conjecture, J. Pure Appl. Algebra 204 (2006) 280-299 MR2184812

[26] M V-P Y Felix, J-C Thomas, Loop homology algebra of a closed manifold arXiv: math.AT/0203137

Department of Mathematics, College of Technology of the City University of New York 300 Jay Street, Brooklyn NY 11201, USA

Department of Mathematics, CW Post Campus of Long Island University 720 Northern Boulevard, Brookville NY 11548, USA

D Sullivan: Department of Mathematics, Graduate Center of the City University of New York 365 Fifth Avenue, New York NY 10016, USA

ttradler@citytech.cuny.edu, mzeinalian@liu.edu, dsullivan@gc.cuny.edu

Received: 21 August $2005 \quad$ Revised: 16 September 2006 